\title{
Dynamics of temporal discrimination
}

\author{
PAULO GUILHARDI and RUSSELL M. CHURCH \\ Brown University, Providence, Rhode Island
}

\begin{abstract}
The purpose of this research was to describe and explain the acquisition of temporal discriminations, transitions from one temporal interval to another, and asymptotic performance of stimulus and temporal discriminations. Rats were trained on a multiple cued interval (MCI) procedure with a head entry response on three signaled fixed-interval schedules of reinforcement (30,60, and 120 sec). They readily learned the three temporal discriminations, whether they were presented simultaneously or successively, and they rapidly adjusted their performance to new intervals when the intermediate interval was varied daily. Although exponential functions provided good descriptions of many measures of temporal discrimination, different parameter values were required for each measure. The addition of a linear operator to a packet theory of timing with a single set of parameters provided a quantitative process model that fit many measures of the dynamics of temporal discrimination.
\end{abstract}

The purpose of this article is to describe and explain the dynamics of temporal discrimination. This includes the original acquisition of a temporal discrimination, transitions from one temporal interval to another, and asymptotic performance on a temporal discrimination.

Although a great deal is known about the asymptotic performance on temporal discrimination tasks, knowledge about the dynamics of temporal discrimination is limited. Some analyses of the acquisition of a temporal discrimination have focused on the dynamics of initial acquisition (Ferster \& Skinner, 1957; Kirkpatrick \& Church, 2000a; Machado \& Cevik, 1998). Other analyses have focused on transition effects - changes in the performance produced by changes in the fixed interval (Higa, 1997; Higa, Thaw, \& Staddon, 1993; Lejeune, Ferrara, Simons, \& Wearden, 1997; Meck, Komeily-Zadeh, \& Church, 1984). The analysis of initial acquisition has not been integrated with the analysis of transition effects. In addition, the analysis of temporal discrimination has not been integrated with the analysis of stimulus discrimination. In fact, many procedures for temporal performance do not include stimulus discrimination (Ferster \& Skinner, 1957; Lejeune et al., 1997; Machado \& Cevik, 1998).

In a review of studies of the acquisition of instrumental responses, Mackintosh (1974) found that the form of the learning curve relating various measures of performance to the amount of training depended on the experimental procedures and the dependent variable used as a measure

This research was supported by National Institute of Mental Health Grant MH44234 to Brown University. The raw data (time of occurrence of each response and reinforcer on each session for each rat) are available at http://www.brown.edu/Research/Timelab. This makes it possible for others to examine alternative dependent variables and to evaluate quantitative theories of timing and conditioning (Church, 2002). Correspondence concerning this article should be addressed to P. Guilhardi, Department of Psychology, Box 1853, Brown University, Providence, RI 02912 (e-mail: paulo_guilhardi@brown.edu). of learning. He concluded that, without more understanding of these factors, "the quest for a typical or true learning curve will be of questionable value" (p. 150). These concerns are still present, and they apply also to temporal learning.

Temporal discrimination is sometimes reported to occur relatively slowly - that is, to require many sessions of training (Ferster \& Skinner, 1957; Schneider, 1969). It has also been reported to occur rapidly - that is, to occur within a session (Higa, 1997; Higa et al., 1993; Lejeune et al., 1997) or even at an optimal rate (Gallistel, Mark, King, \& Latham, 2001). The speed of learning of a temporal discrimination undoubtedly depends on the timing task, previous experience, and the criterion of learning. In skilled performance of any sort, there may be evidence of learning that occurs almost immediately and of substantial further learning with additional training.

For the study of the dynamics of temporal discrimination, it is desirable to use a procedure that produces rapid learning of distinct behavior by individual animals for different temporal intervals between stimuli and reinforcers. In the present experiment, a multiple cued interval (MCI) procedure was used in which rats were trained on three different cued fixed-interval (FI) schedules of reinforcement. In this MCI procedure, a cycle consisted of an interval of time without a stimulus and an interval of time with a stimulus; the first response after the stimulus had been on for a fixed number of seconds was followed by the delivery of a reinforcer and the termination of the stimulus. This cycle was repeated throughout a session and for many sessions. With an MCI procedure, there are multiple cues and multiple intervals; in the present experiment, there were three cues (white noise, a houselight, and a clicker) for three FIs (30, 60, and $120 \mathrm{sec})$.

In an MCI procedure, changes in different dependent variables as a function of training serve as indices of the acquisition of a stimulus discrimination (differential responding during the presence and absence of the stimu- 
lus), within-interval temporal discrimination (differential responding early and late during the stimulus), and between-interval temporal discrimination (differential responding in intervals of different duration).

Many different dependent measures have been used to describe temporal discriminations. In some cases, a measure has been based on a description of the pattern of results observed in a cumulative record (Cumming \& Schoenfeld, 1958; Ferster \& Skinner, 1957; Machado \& Cevik, 1998; Schneider, 1969). In other cases, a quantitative index has been calculated. These include the postreinforcement pause or waiting time (Dukich \& Lee, 1973; Higa, 1997), the time to the fourth response (Dukich \& Lee, 1973), the quarter-life (Gollub, 1964), the temporal discrimination ratio (Kirkpatrick \& Church, 2000a, 2000b), the curvature index (Fry, Kelleher, \& Cook, 1960), the average response rate (Catania \& Reynolds, 1968), the peak time (Catania \& Reynolds, 1968; Lejeune et al., 1997; Roberts, 1981), and the time of rate change (Church, Meck, \& Gibbon, 1994; Schneider, 1969). Some of the steady state measures of temporal discriminations are correlated (Dukich \& Lee, 1973; Gollub, 1964), but systematic differences in the measures as a function of training would indicate that they are not redundant.

During discriminative FI schedules of reinforcement, such as the MCI procedure, four qualitative features of behavior emerge.

1. Overall response rate changes. The overall response rate increases as a function of sessions of training (Spence, 1956).

2. Stimulus discrimination. The response rate during the stimulus is higher than the response rate during the absence of the stimulus (Skinner, 1938). A ratio of these response rates may be used as a measure of stimulus discrimination.

3. Within-interval temporal discrimination. The response rate is higher at the end than at the beginning of the stimulus (Ferster \& Skinner, 1957). A ratio of these response rates may be used as a measure of the withininterval temporal discrimination. On individual cycles, the response rate during the stimulus is often characterized by a break-run pattern of responding, which is a period of low-rate responding followed by a period of high-rate responding (Church et al., 1994; Cumming \& Schoenfeld, 1958; Schneider, 1969). The response rate during the stimulus, averaged over many cycles, increases as a function of time since stimulus onset, reaching its maximum near the end of the FI. These averaged response gradients are often ogival in shape.

4. Between-interval temporal discrimination. Some of the properties of the break-run gradients of individual cycles and the ogival response gradients averaged over many cycles indicate that animals also discriminate different intervals. The time from stimulus onset to the first response (initial pause) is positively related to the FI duration (Ferster \& Skinner, 1957) and is approximately proportional to the interval duration (Catania, 1970; Innis \& Staddon, 1971). The time at which response rate changes from a low to a high rate is also related to the interval duration
(Cumming \& Schoenfeld, 1958; Schneider, 1969). After extensive training, when individual trials are averaged, the maximum response rate and the slope of the response gradient are inversely related to interval duration (Catania $\&$ Reynolds, 1968). The differences between the response gradients produced by different intervals may be used as a measure of between-interval temporal discrimination.

The Results section will describe an empirical approach to temporal discrimination learning in which simple exponential equations will provide a good description of the acquisition of a large number of dependent variables. Because no simple rules were identified for the differences in the best-fitting parameters of the exponential equations that fit the different dependent variables, this direct approach will be considered to be simply curve fitting.

The Discussion section describes a packet theory of timing that, with the procedure as an input, predicts the time of occurrence of stimuli, responses, and reinforcements. Because the same model with the same parameters provides a good description of multiple dependent measures, this indirect theoretical approach will be considered to be an explanation of the behavior.

\section{METHOD}

\section{Animals}

Twenty-four male Sprague Dawley rats (Taconic Laboratories, Germantown, NY) were housed individually in a colony room on a 12:12-h light:dark cycle (lights off at 8:30 a.m.). Dim red lights provided illumination in the colony room and the testing room. The rats were fed a daily ration that consisted of 45 -mg Noyes pellets (Improved Formula A), which were delivered during the experimental session, and an additional $15 \mathrm{~g}$ of FormuLab 5008 food given in the home cage after the daily sessions. Water was available ad lib in both the home cages and the experimental chambers. The rats arrived in the colony at 35 days of age and were handled daily until the onset of the experiment. Training began when they were 67 days old.

\section{Apparatus}

The 12 chambers $(25 \times 30 \times 30 \mathrm{~cm})$ were located inside ventilated, noise-attenuating boxes $(74 \times 38 \times 60 \mathrm{~cm})$. Each chamber was equipped with a food cup and a water bottle. Three stimuli, referred to as noise, light, and clicker, were generated from modules from Med Associates (St. Albans, VT). The noise was a 70-dB white noise, with an onset rise time and termination fall time of $10 \mathrm{msec}$, that was generated by an audio amplifier (Model ANL-926). The light was a diffused houselight (Model ENV-227M) rated to illuminate the entire chamber over 200 Lux at a distance of 3 in. The clicker (Model ENV-135M) was a small relay mounted on the outside of the chamber that was used to produce an auditory click at a rate of one per second. A pellet dispenser (Model ENV-203) delivered 45-mg Noyes (Improved Formula A) pellets into the food cup on the front wall. Each head entry into the food cup was detected by an LED photocell. A water bottle was mounted outside the chamber; water was available through a tube that protruded through a hole in the back wall of the chamber. Two Gateway Pentium III/500 computers running the MED-PC for Windows Version 1.15 using Medstate Notation Version 2.0 (Tatham \& Zurn, 1989) controlled experimental events and recorded the time at which events occurred with 2-msec resolution.

\section{Procedure}

The experimental sessions consisted of 60 cycles or $150 \mathrm{~min}$, whichever came first. The animals were trained on the MCI proce- 
dure, in which each cycle consisted of a 20 -sec period with discriminative stimuli off, followed by a period with a discriminative stimulus on. Food was primed at the end of an FI. Immediately after the next head entry into the food cup, measured as the time of breaking a photobeam in the food cup, food was delivered, the discriminative stimulus was turned off, and the next cycle began.

Phase 1 (Sessions 1-30) was baseline training with three intervals. During baseline, all the animals were trained for 30 sessions under 30-, 60-, and 120-sec FI schedules of reinforcement differentially signaled by noise, a light, or a clicker. For example, a particular rat might have a 30 -sec interval signaled by noise, a 60 -sec interval signaled by the light, and a 120 -sec interval signaled by the clicker. The assignment of stimuli to intervals was counterbalanced.

Twelve rats were randomly assigned to the blocked condition, and the other 12 rats were assigned to the simultaneous condition. Training for the blocked condition consisted of 10 sessions with one interval, then 10 sessions with a second interval, and then 10 sessions with the third interval. The six possible orders of the three intervals were counterbalanced, with 2 rats randomly assigned to each of the six possible orders of the three intervals $(30,60$, and $120 \mathrm{sec})$. Training in the simultaneous condition consisted of 30 sessions in which one of the three possible intervals was presented randomly with equal probability on each cycle of each session. After 30 sessions of training, the rats from both conditions had received approximately 600 cycles of each interval.

Phase 2 (Sessions 31-66) was training with daily changes in the intermediate interval. During this phase, there were cued intervals of 30 and $120 \mathrm{sec}$ (with the same stimuli for each rat as that used in Phase 1) and one of nine intervals with the stimulus previously used for the 60 -sec interval in Phase 1 . The nine possible middle intervals were distributed between 30 and $120 \mathrm{sec}$ in an approximately logarithmic manner $(30.00,35.68,42.43,50.54,60.00,71.35,84.85$, 100.90 , and $120.00 \mathrm{sec}$ ). As in Phase 1, the three intervals were randomly presented during every session.

The 12 rats in each condition (the blocked and the simultaneous conditions) were randomly partitioned into two groups of 6 rats in two conditions that differed in the pattern in which the middle interval changed every session. For the ramp condition, the middle intervals changed in a ramped order. The rats started with a 60 -sec inter$\mathrm{val}$; half continued with an ascending order of intervals, whereas the other half continued with a descending order. When the minimum interval $(30 \mathrm{sec})$ was reached, this interval was repeated once, and the interval increased on successive sessions to the maximum interval $(120 \mathrm{sec})$; when the maximum interval $(120 \mathrm{sec})$ was reached, this interval was repeated once, and the intervals decreased on successive sessions to the minimum interval $(30 \mathrm{sec})$. For the random condition, the middle interval on each of nine sessions consisted of a random ordering of the nine possible intervals. This process was repeated four times, so that each of the nine intervals was trained for a total of four sessions.

\section{RESULTS}

\section{Phase 1: Baseline (Sessions 1-30)}

Response rate during the stimulus. The mean response rate during the stimulus (Figure 1) was approximately the same for the three intervals in the first block of 20 cycles for the simultaneous condition (mean of 11.7 responses per minute; top panel) and for the blocked condition (mean of 9.5 responses per minute; bottom panel). With training, this measure increased exponentially toward different asymptotic levels of $45.8,43.7$, and 34.5 responses per minute for the simultaneous condition and of $46.5,40.2$, and 28.0 responses per minute for the blocked condition, for the FI 30-, 60-, and 120-sec intervals, respectively.
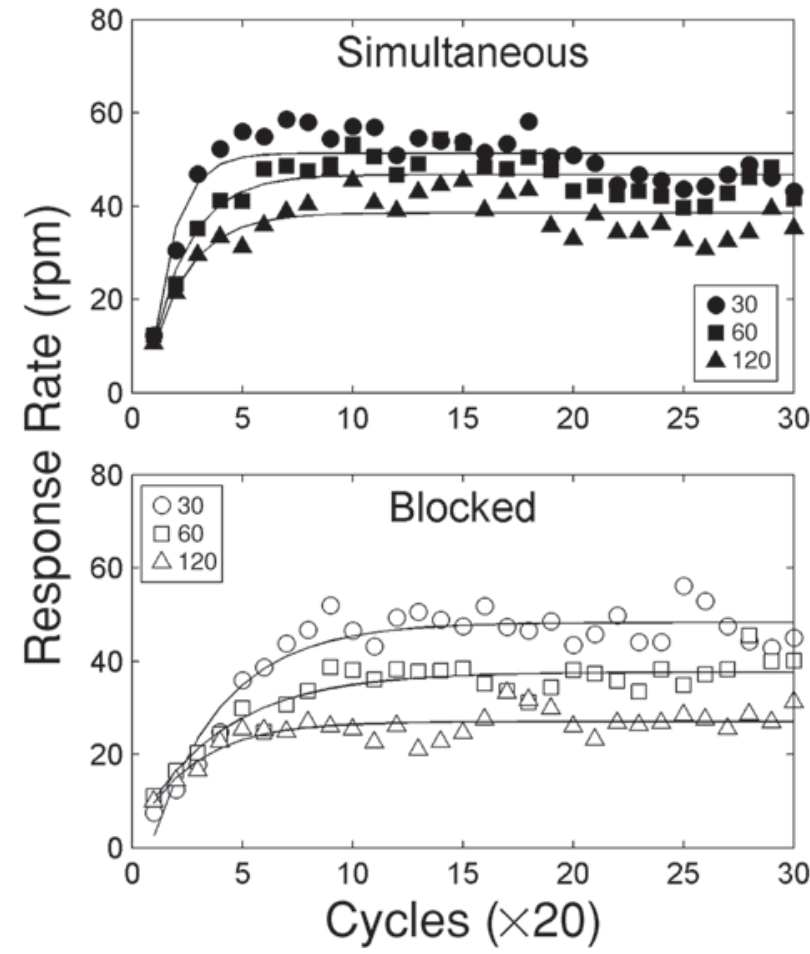

Figure 1. Response rate during the stimulus as a function of blocks of 20 cycles is shown for the simultaneous condition (top panel) and the blocked condition (bottom panel) for the 30-, 60-, and 120 -sec intervals. The thin lines are the best-fitting exponential equations.

The thin lines in Figures 1, 2, and 3 are the best-fitting three-parameter exponential equations (Equation 1):

$$
y=(c-a)\left(1-e^{-b n}\right)+a,
$$

where $a$ is the intercept, $b$ is the scale, and $c$ is the asymptote. The variable $n$ was either session number or cycle number (as specified in the text). A nonlinear search algorithm (nlinfit) that minimized the sum of squares was used for the estimation of the parameters $a, b$, and $c$. The scale of the functions $(b)$ was used as a measure of the speed of learning. The best-fitting equation of this form was found for each rat in each condition and also for the mean across rats. The figures show the best-fitting equation to the fit of the mean performance. The statistical conclusions shown in Table 1 were based on the best-fitting parameters for the individual rats. The goodness-of-fit measure $\left(\omega^{2}\right)$ for a particular dependent variable was the ratio of the variance accounted for by the exponential functions for each of the intervals relative to the total variance of the data across intervals.

Table 1 provides the estimates of the first 20 cycles, the learning rate, and the last 20 cycles for each of the dependent variables. The statistical significance of the effect of the fixed interval $(30,60$, and $120 \mathrm{sec})$ on the dependent variables is shown by the symbols for both the simultaneous and the blocked conditions. Some of the statistically 
Table 1

Estimates of Goodness of Fit of Exponential Functions to the Five Summary Dependent Measures of Temporal Discrimination for the Simultaneous (S) and Blocked (B) Conditions

\begin{tabular}{|c|c|c|c|c|c|c|c|c|c|c|c|}
\hline \multirow[b]{2}{*}{ Dependent Measure } & \multirow[b]{2}{*}{ Condition } & \multicolumn{3}{|c|}{ First 20 Cycles } & \multicolumn{3}{|c|}{ Learning Rate } & \multicolumn{3}{|c|}{ Last 20 Cycles } & \multirow{2}{*}{$\begin{array}{l}\text { Goodness } \\
\text { of Fit }\left(\omega^{2}\right)\end{array}$} \\
\hline & & FI 30 & FI 60 & FI 120 & FI 30 & FI 60 & FI 120 & FI 30 & FI 60 & FI 120 & \\
\hline \multirow[t]{2}{*}{ Time of median response (sec) } & $\mathrm{S}$ & 19.46 & 33.33 & $60.24^{* * *}$ & 0.13 & 0.24 & $0.22^{*}$ & 25.66 & 49.66 & $98.18^{* * *}$ & .998 \\
\hline & $\mathrm{B}$ & 19.45 & 31.37 & $57.92^{* * *}$ & 0.05 & 0.15 & $0.15^{\dagger}$ & 25.27 & 49.31 & $98.42^{* * *}$ & .995 \\
\hline \multirow[t]{2}{*}{ Time of response rate change (sec) } & $\mathrm{S}$ & 15.32 & 30.14 & $47.20^{* * *}$ & 0.07 & 0.05 & $0.17^{\dagger}$ & 20.67 & 41.12 & $76.92^{* * *}$ & .996 \\
\hline & $\mathrm{B}$ & 15.94 & 26.61 & $52.05^{* * *}$ & 0.01 & 0.05 & $0.04^{\dagger}$ & 19.86 & 40.7 & $81.19^{* * *}$ & .989 \\
\hline \multirow[t]{2}{*}{ Time of first response (sec) } & $\mathrm{S}$ & 8.81 & 8.41 & $8.70^{\dagger}$ & 0.13 & 0.17 & $0.15^{\dagger}$ & 18.4 & 30.36 & $50.15^{* * *}$ & .976 \\
\hline & $\mathrm{B}$ & 7.78 & 6.18 & $4.73^{*}$ & 0.03 & 0.13 & $0.06^{\dagger}$ & 16.56 & 26.29 & $59.71^{* * *}$ & .936 \\
\hline \multirow{2}{*}{ Response rate (rpm) } & $\mathrm{S}$ & 10.95 & 18.74 & $13.91^{* * *}$ & 0.95 & 0.08 & $0.21^{* * *}$ & 51.3 & 7.85 & $1.96^{* * *}$ & .977 \\
\hline & $\mathrm{B}$ & 2.67 & 17.04 & $14.23^{* *}$ & 0.31 & 0.02 & $0.10^{\dagger}$ & 48.29 & 6.52 & $1.25^{* * *}$ & .974 \\
\hline \multirow[t]{2}{*}{ Temporal discrimination ratio } & $\mathrm{S}$ & 0.52 & 0.51 & $0.46^{\dagger}$ & 0.57 & 0.61 & $0.56^{\dagger}$ & 0.99 & 0.98 & $0.99^{\dagger}$ & .985 \\
\hline & B & 0.52 & 0.43 & $0.37^{* *}$ & 0.17 & 0.26 & $0.28^{\dagger}$ & 1.00 & 0.97 & $0.98^{\dagger}$ & .961 \\
\hline
\end{tabular}

Note-The parameters of the exponential functions were the start (based on the first 20 cycles), the end (based on the last 20 cycles), and the learning rate (based on the scale of the best-fitting exponential function; see Equation 3). ${ }^{*} p<.05 .{ }^{* *} p<.01 .{ }^{* * *} p<.001 . \quad{ }^{\dagger} p>.05$.

significant differences observed during the first 20 cycles may have represented learning during these cycles; some of the significant differences may have been due to the large number (180) of comparisons made; and some may have been based on consistent differences that were of small magnitude. Most of the statistically significant differences are consistent with the large effects evident in the figures.

Stimulus discrimination ratio. The stimulus discrimination ratio provided a comparison of the response rate during the middle of a stimulus with the response rate prior to the stimulus. The measure of the response rate during the stimulus was a 5 -sec interval centered at the middle of the stimulus $\left(r_{\mathrm{m}}\right)$; the measure of the response rate prior to the stimulus was a 5 -sec interval that ended at stimulus onset $\left(r_{\mathrm{p}}\right)$. The stimulus discrimination ratio was defined as $r_{\mathrm{m}} /\left(r_{\mathrm{p}}+r_{\mathrm{m}}\right)$, with .5 indicating no stimulus discrimination and 1.0 indicating perfect stimulus discrimination.

The stimulus discrimination ratio increased for all conditions from a level indicating little or no stimulus discrimination (.5) to one indicating nearly complete stimulus discrimination (1.0; see the top panels of Figure 2). The rats in the simultaneous conditions learned the stimulus discrimination more rapidly than did the rats in the blocked conditions when the stimulus discrimination ratio was plotted as a function of cycles $[F(1,22)=7.0, p=$ $.014]$, but at about the same rate when it was plotted as a function of sessions $[F(1,22)=1.8$, n.s. $]$.

Temporal discrimination ratio. The temporal discrimination ratio provided a comparison of the response rate at the end of an interval with the response rate at the beginning of the interval. The measure of response rate at the beginning of an interval $\left(r_{\mathrm{b}}\right)$ was an interval that was two fifteenths of the stimulus duration that began at stimulus onset; the measure of the response rate at the end of the stimulus $\left(r_{\mathrm{e}}\right)$ was an interval that was two fifteenths of the stimulus duration that ended at the time that food was available. The temporal discrimination ratio was defined as $r_{\mathrm{e}} /\left(r_{\mathrm{b}}+r_{\mathrm{e}}\right)$, with .5 indicating no temporal discrimination and 1.0 indicating perfect temporal discrimination.

The temporal discrimination ratio increased for all conditions from a level indicating little or no temporal discrimination (.5) to one indicating nearly complete tem- poral discrimination (1.0; see the middle panels of Figure 2). The rats in the simultaneous conditions learned the temporal discrimination more rapidly than did the rats in the blocked conditions when the temporal discrimination ratio was plotted as a function of cycles $[F(1,22)=44.9$, $p<.001]$, but at about the same rate when it was plotted as a function of sessions $[F(1,22)=0.3$, n.s.]. The mean stimulus and temporal discrimination ratios for the rats in the six conditions (simultaneous and blocked with $30-, 60-$, and 120 -sec intervals) are shown as a function of sessions in the bottom panel of Figure 2. The stimulus and temporal discrimination ratios were similar, but the scale of the stimulus discrimination ratio as a function of sessions was more rapid than the scale of the temporal discrimination ratio $[F(1,23)=5.7, p=.026]$. Although the magnitude of the effect was small, the scale of the stimulus discrimination ratio was steeper than the scale of the temporal discrimination ratio for 22 of the 24 rats.

Time of the median response. The time of the median response was defined as the latency from the onset of a stimulus to the time of the median response in a cycle. It initially differed as a function of interval $[F(2,22)=$ $587.0, p<.001$, and $F(2,9)=116.6, p<.001$, for the simultaneous and the blocked conditions, respectively; see Figure 3, top panel]. The functions gradually increased exponentially toward different asymptotic levels [of 25 , 49, and $99 \mathrm{sec}$ for FI 30, FI 60, and FI $120 \mathrm{sec}$, respectively; $F(2,22)=5,505.1, p<.001$, for the simultaneous condition, and $F(2,9)=404.3, p<.001$, for the blocked condition].

Time of the maximum rate change. The time of the maximum rate change during a cycle is defined as the time $\left(t_{1}\right)$ that maximizes the following equation:

$$
A=t_{1}\left(r-r_{1}\right)+t_{2}\left(r_{2}-r\right),
$$

in which $r_{1}$ is the response rate prior to $t_{1}, r_{2}$ is the response rate after $t_{1}$, and $r$ is the mean response rate. Also, $t_{1}$ is the duration prior to $t_{1}$, and $t_{2}$ is the duration from $t_{1}$ to food delivery. An exhaustive search at the times of each response during a cycle determines the value of $t_{1}$ that maximizes the area, $A$. 

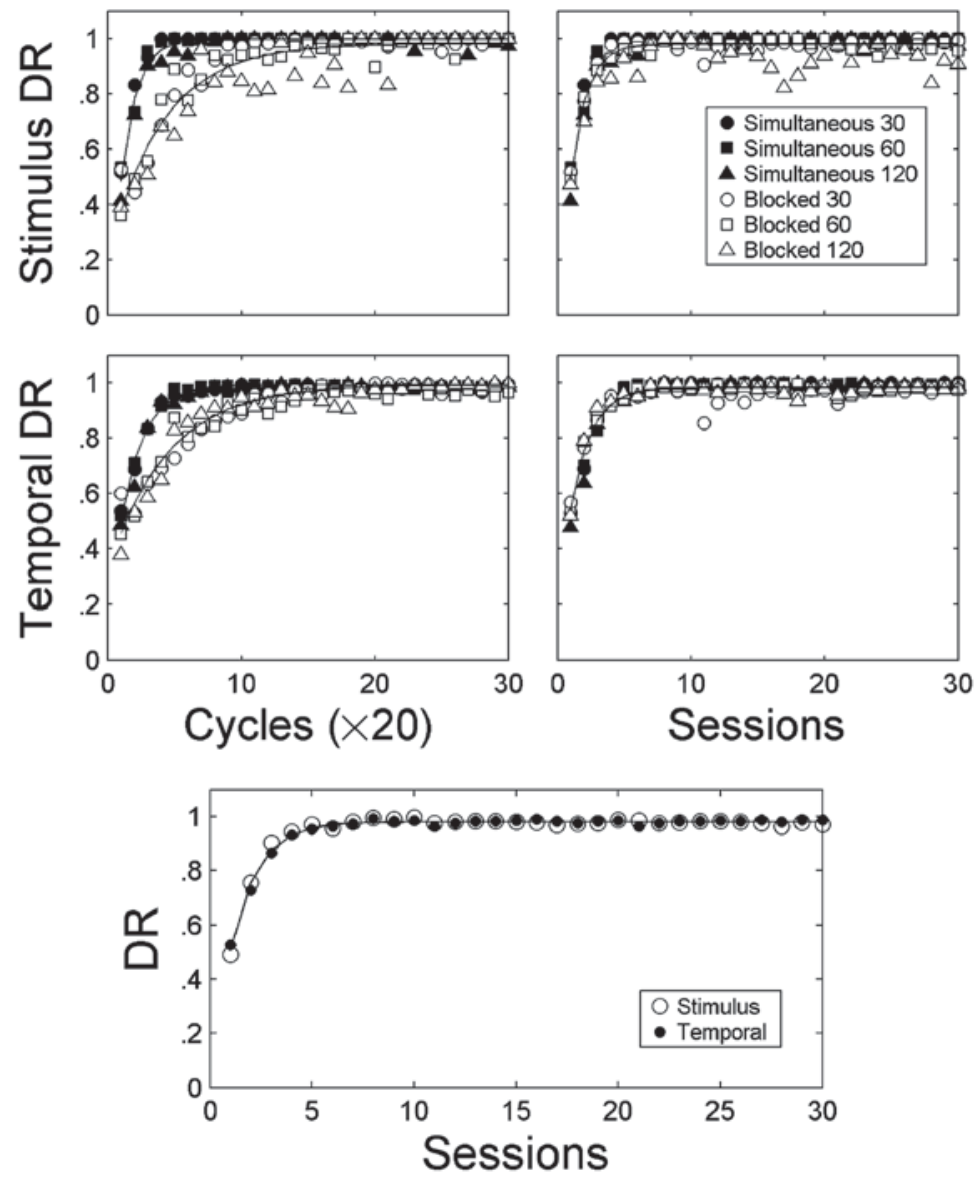

Figure 2. Top panels: Stimulus discrimination ratio (DR) of the simultaneous and blocked conditions at three intervals $(30,60$, and 120 sec). Middle panels: Temporal DR of the simultaneous and blocked conditions at three intervals (30, 60 , and $120 \mathrm{sec}$ ). These dependent variables are plotted as a function of blocks of 20 cycles (left panels) and as a function of sessions (right panels). Bottom panel: A comparison of the stimulus DR and the temporal DR as a function of sessions. The thin lines are the best-fitting exponential equations.

The values of $t_{1}$ as a function of blocks of 20 cycles is shown in the second panel of Figure 3. In the simultaneous condition, the initial $t_{1}$ was positively related to the number of cycles $[F(2,22)=182.2, p<.001]$; the asymptotic value of $t_{1}$ was negatively related to the interval $[F(2,22)=1,616.7, p<.001]$, but the scales were equivalent $[F(2,22)=1.3$, n.s.]. The results from the blocked condition were similar to those from the simultaneous condition.

Time of the first response. The time of the first response was defined as the latency from the onset of a stimulus to the first response in a cycle. On the first block of 20 cycles, it was approximately the same for the three intervals on the first block of 20 cycles - a mean of $11.4 \mathrm{sec}$ (Figure 3, third panel). With training, this measure increased exponentially toward different asymptotic levels (of 18.7, 30.6, and $56.9 \mathrm{sec}$ for FI 30, FI 60, and FI $120 \mathrm{sec}$, respectively). The asymptote of the best-fitting exponential functions relating time of the first response to cycles were different for the intervals of 30,60 , and $120 \mathrm{sec}[F(2,22)=98.6, p<.001$, and $F(2,9)=20.4$, $p<.001$, for the simultaneous and the blocked conditions, respectively]. The measure of the speed of learning was similar at all three intervals $[F(2,22)=2.2$, n.s., and $F(2,9)=1.0$, n.s., for the simultaneous and the blocked conditions, respectively].

Response rate at comparable intervals. The response rate at comparable intervals was defined as the mean response rate during the first $30 \mathrm{sec}$ from stimulus onset. Because the FI durations used in the present experiment ranged from 30 to $120 \mathrm{sec}$, all the animals had an equal opportunity to respond in the first $30 \mathrm{sec}$ since stimulus onset on every cycle. The mean response rate had a pattern of results similar to the time of the first response (Figure 3, fourth panel). It was approximately the same for the three intervals on the first block of 20 cycles (average of 12.5 responses per minute). With training, this measure increased or decreased exponentially toward an 


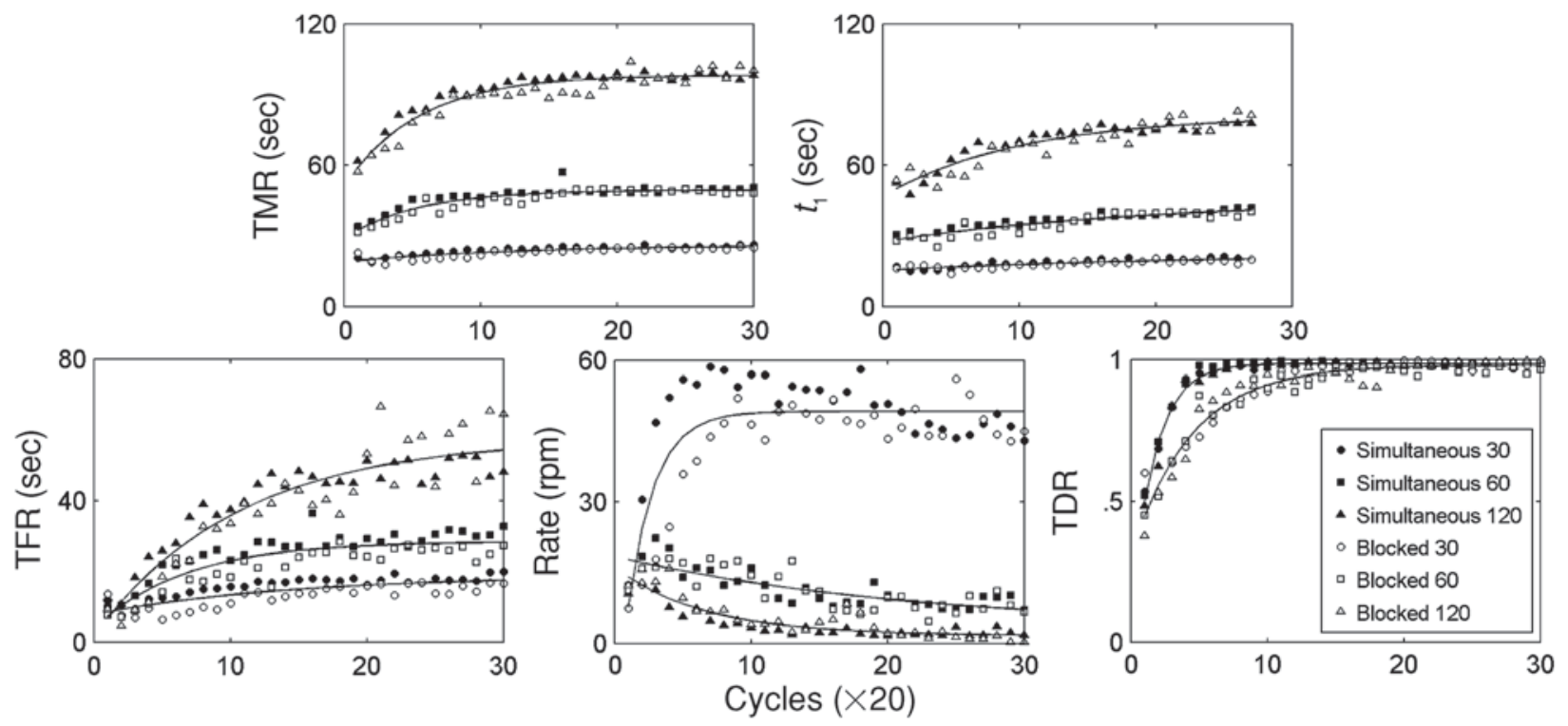

Figure 3. The change in five measures of timing as a function of blocks of 20 cycles for the simultaneous and blocked conditions at three intervals $(30,60$, and 120 sec). The thin lines are the best-fitting exponential equations. The measures are the time of median response (TMR; first panel), the time of transition ( $t_{1}$; second panel), the time of first response (TFR; third panel), the mean response rate in responses per minute (fourth panel), and the temporal discrimination ratio (TDR; fifth panel). (The mean response rate was calculated during the first 30 sec of the stimuli under all conditions.)

asymptotic level $(50.6,12.2$, and 2.1 responses per minute for FI 30, FI 60, and FI $120 \mathrm{sec}$, respectively). The mean response rate at comparable intervals was inversely related to the interval duration and also was related to the distance from the response rate at the beginning of training to the asymptotic response rate level. The asymptotes were different for the intervals of 30,60 , and $120 \mathrm{sec}[F(2,22)=$ $144.7, p<.001$, and $F(2,9)=405.5, p<.001$, for simultaneous and blocked conditions, respectively]. The mean scales for the blocked condition were $0.32,0.32$, and .08 for 30,60 , and $120 \mathrm{sec}$, respectively, and were not related to interval duration $[F(2,9)=0.6$, n.s.]. The mean scales for the simultaneous condition were $0.93,0.10$, and 0.22 for 30,60 , and $120 \mathrm{sec}$, respectively, and were related to interval duration $[F(2,22)=21.8, p<.001]$. The speed of learning for this measure was not affected by the interval duration for the blocked condition but was affected for the simultaneous condition.

Temporal discrimination ratio. The temporal discrimination ratio as a function of cycles is replotted in the fifth panel of Figure 3 so that it can be readily compared with the other four measures of temporal discrimination. This measure of temporal discrimination began at approximately the same level for each fixed interval and ended at approximately the same level for each interval.

Two of the measures of temporal discrimination began and ended at different levels for each interval (see Figure 3, panels 1 and 2); two of the measures began at approximately the same level and ended at different levels for each interval (panels 3 and 4); and one of the measures began and ended at approximately the same level for each interval (panel 5).
Temporal gradients. The absolute temporal gradients were the mean response rates (in responses per minute) as a function of time since stimulus onset (in seconds). These gradients are shown for the 30-, 60-, and 120-sec FIs for the simultaneous and the blocked groups (Figure 4, top left and right panels, respectively). The relative temporal gradients were the mean response rates as proportions of the maximum rates. The ogives that best fit these response gradients were calculated on the basis of Equation 3:

$$
y=\frac{c}{1+e^{-(x-a) / b}} .
$$

In all eight ogives shown in Figure 4, the minimum rate was set to 0 ; in the two bottom panels of Figure 4, the maximum was set to 1.0. A nonlinear search algorithm that minimized the sum of squares was used for the estimation of the parameters $a, b$, and $c$, which served as measures of temporal discrimination. This was done with the nlinfit function of MATLAB (The MathWorks, Natick, MA).

1. The parameter $a$ is an estimate of the center (the time at which the response rate reached half of the way to its estimated maximum response rate).

2. The parameter $b$ is an estimate of the scale of the function, a measure of the precision of timing.

3 . The parameter $c$ is an estimate of the maximum response rate of the function.

The temporal gradients shown in Figure 4 were based on the response rate averaged over the last 300 cycles. These were the last 15 sessions for the simultaneous condition and Sessions 6-10, 16-20, and 26-30 for the blocked condition. The gradients were related to the duration of the fixed interval in both training conditions (Figure 4, 

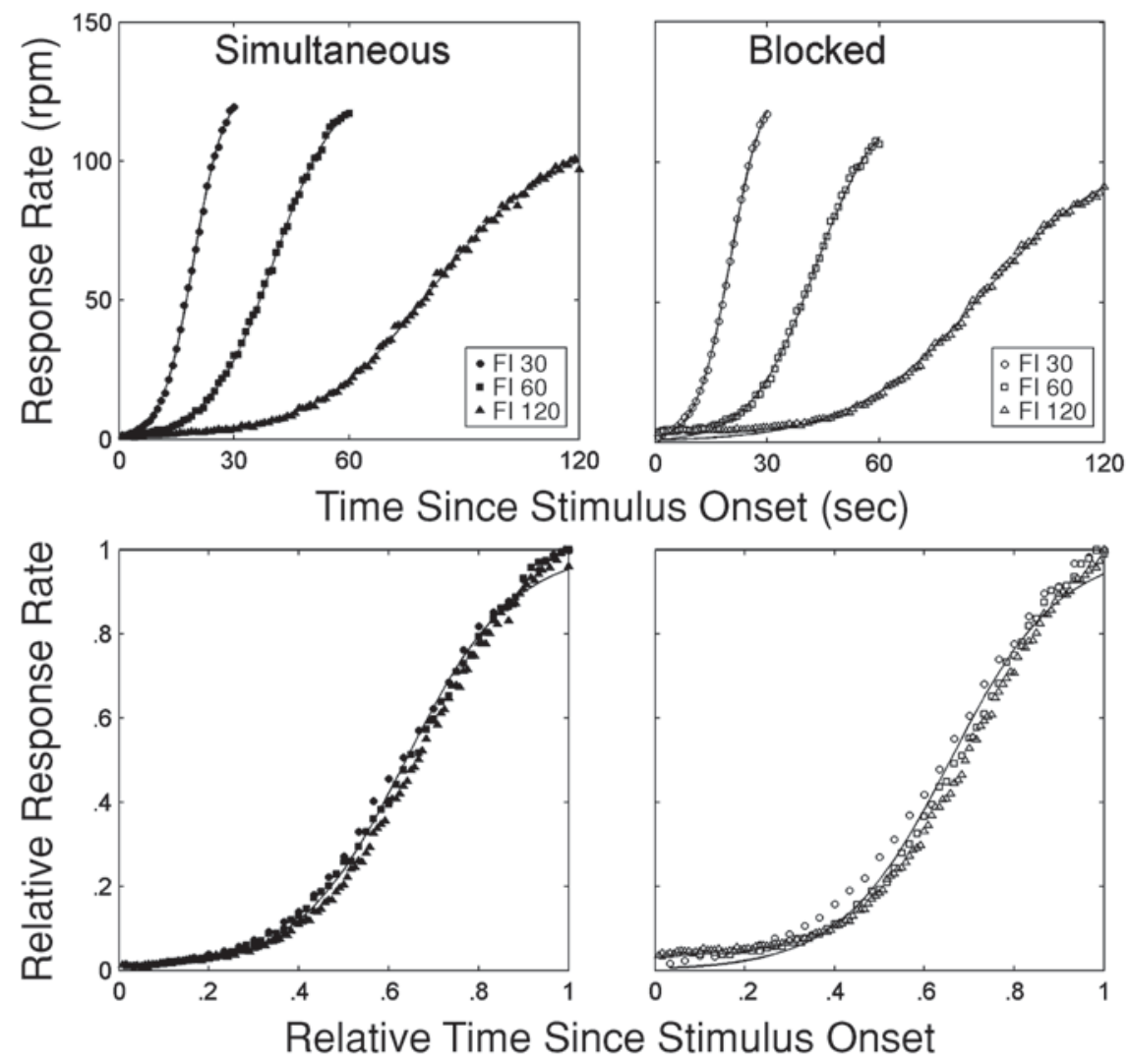

Figure 4. Response rate as a function of time since stimulus for the three fixed intervals (FIs). The left panels show gradients of responding for the simultaneous conditions, and the right panels show the gradients for the blocked conditions; the top panels show the gradients for the time in seconds, and the bottom panels show the gradients for time as a proportion of the interval. The thin lines are the best-fitting ogive functions.

top panels). The relative response gradients, expressed as a function of the proportion of the interval, were similar at all intervals (Figure 4, bottom panels). Such overlap is referred to as superposition or timescale invariance. The median proportion of variance accounted for was .999 , with a range from .995 to .999 .

The similarity is even greater if a 2.25 -sec interval is subtracted from each of the times, on the basis of the assumption that there is a latency to begin timing that is the same at all intervals. There was no relationship between the centers of the ogival functions (Equation 2) and interval duration for the blocked condition $[F(2,22)=0.3$, n.s.] or for the simultaneous condition $[F(2,22)=0.2$, n.s.]. There was also no relationship between the scales of the ogival functions and the interval duration for either the blocked condition $[F(2,22)=1.7$, n.s. $]$ or the simultaneous condition $[F(2,22)=1.6$, n.s. $]$.

The acquisition of the temporal gradients is shown in Figure 5 for Sessions 1-6 and for the last six blocks of 20 cycles. On the first session, the response rate was relatively constant as a function of time since stimulus onset. A flat gradient characterized the performance at all the intervals $(30,60$, and $120 \mathrm{sec})$ and with both the simulta- neous and the blocked conditions. The temporal learning consisted of an increase in response rate late in the interval and a decrease in response rate early in the interval.

On Sessions 16-30 (simultaneous condition) or 6-10 (blocked condition), the response rate increased as a function of time since stimulus onset. This asymptotic gradient was well characterized as an ogive at all the intervals and in both the simultaneous and the blocked conditions (see the description in Figure 4).

\section{Phase 2: Transitions Between Temporal Intervals (Sessions 31-66)}

Figure 6 shows the temporal learning of daily changes in the intermediate interval. In this phase, the two extreme intervals were maintained at 30 and $120 \mathrm{sec}$, whereas the middle interval was changed daily. The figure shows the mean response gradients in the ramp (left panels) and random (right panels) conditions for the first five cycles (top panels), the next five cycles (middle panels), and the last five cycles (bottom panels) of each session. Performance on the two extreme intervals was maintained, but there was a substantial difference in the performance of the rats in the ramp and the random conditions on the middle interval. In 


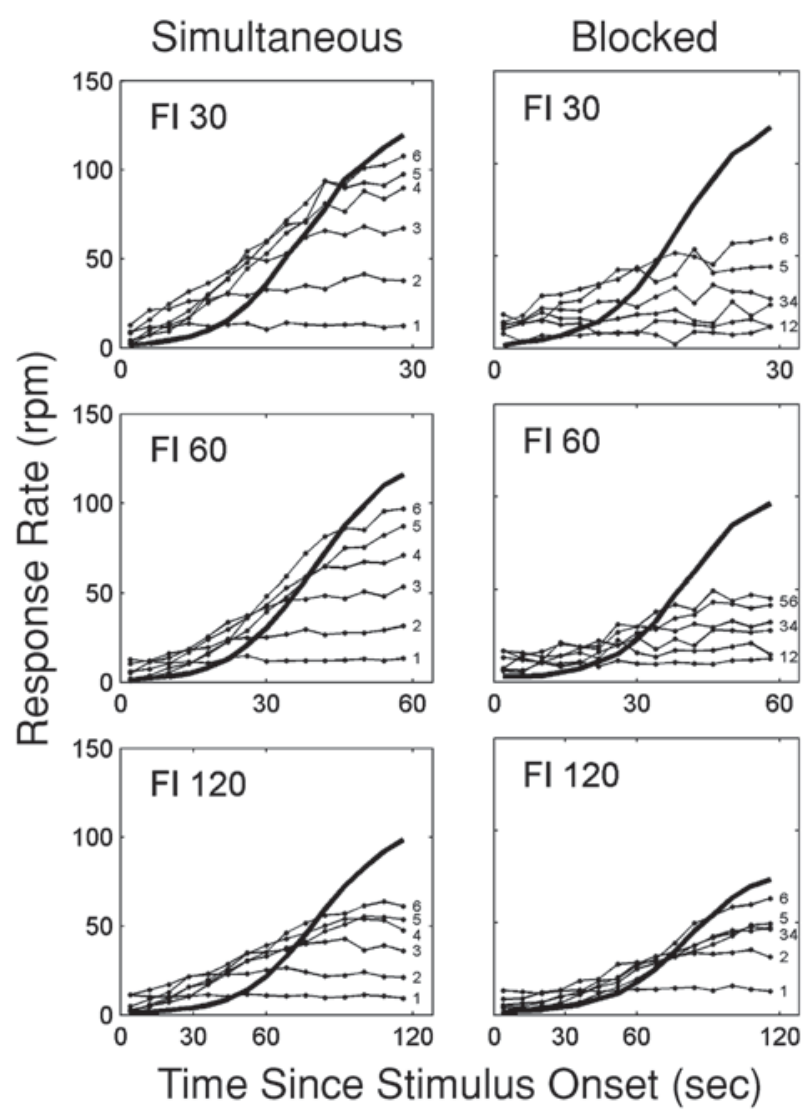

Figure 5. Mean response rate as a function of stimulus onset for the simultaneous condition (left panels) and the blocked condition (right panels) on the 30-, 60-, and 120-sec intervals (top, middle, and bottom panels, respectively). The functions are shown for the first six blocks of 20 cycles (labeled 1-6) and the last six blocks of 20 cycles (thick lines) for the simultaneous and blocked conditions.

the ramp condition, the mean performances on the first and last five cycles of each session were similar. In contrast, in the random condition, the mean performances on the first five cycles of each session were similar, but there was substantial and rapid learning during each session.

\section{DISCUSSION}

The MCI procedure provided a way to study the acquisition and the asymptotic performance of stimulus and temporal discriminations. It also provided a way to study the change in behavior produced by a daily change from one temporal interval to another. With the MCI procedure, rats readily learned three stimulus discriminations (to the light, noise, and clicker) and three temporal discriminations (of 30, 60, and $120 \mathrm{sec}$ ). They learned these discriminations about equally well whether they were all trained simultaneously (in the simultaneous condition) or whether they were trained successively (in the blocked condition).

\section{Initial Acquisition of a Temporal Discrimination}

Initial acquisition was characterized by an increase in response rate during the stimulus (Figure 1), in the stimulus discrimination ratio (Figure 2), in the time of the first response, in the time of the median response, in the time of transition from a low to a high rate, and in the response rate at comparable times since stimulus onset (Figure 3). The response gradient also changed as a function of sessions (Figure 5). All of these changes occurred in the same sessions.

Two features of temporal discrimination were learned during initial acquisition: within-interval and betweenintervals temporal discrimination. Within-interval temporal discrimination started at approximately .5 (no discrimination) and reached almost 1.0 (complete discrimination). The within-interval temporal discrimination ratio for the blocked and simultaneous conditions superposed when plotted as a function of sessions, but not when plotted as a function of cycles trained. This result suggests that the within-interval temporal discrimination (low responding in the beginning of the interval and high responding at the end of the interval) is a common learned feature that occurs independently of the interval being trained. The between-intervals temporal discrimination also started at a no-discrimination level and reached different levels for different intervals. The rate of initial acquisition was similar for intervals of different duration.

The time of median response described the acquisition of both within-interval and between-intervals temporal discrimination. The analysis based solely on time of median response, however, does not provide information about the rates of responding prior to and followed by the median response. Asymptotic temporal gradients superposed when plotted in relative scales supporting the scalar model of timing processes (Gibbon, 1977, 1991; Gibbon $\&$ Church, 1990). Therefore, the centers and scales were proportional to interval duration.

The time of response rate change $\left(t_{1}\right)$ measure started at about half of the interval duration (no discrimination) and reached asymptote at about two thirds of the interval duration. This asymptotic finding is consistent with results of Schneider (1969) and Dukich and Lee (1973), who also found that the transition between the period of no responding and the period of responding (break-run pattern) in an FI schedule of reinforcement occurred at about two thirds of the interval duration after extensive training.

Thus, different summary measures provided evidence of the acquisition of within-interval and between-intervals temporal discrimination. Some measures (such as the stimulus discrimination ratio) did not provide evidence of temporal discrimination. Other measures (such as the temporal discrimination ratio) provided evidence for within-interval temporal responding by comparing the response rate at the end of the stimulus with the response rate at the beginning of the stimulus. Still other measures (such as the response rate during the stimulus, the time of the first response, and the response rate during the first 


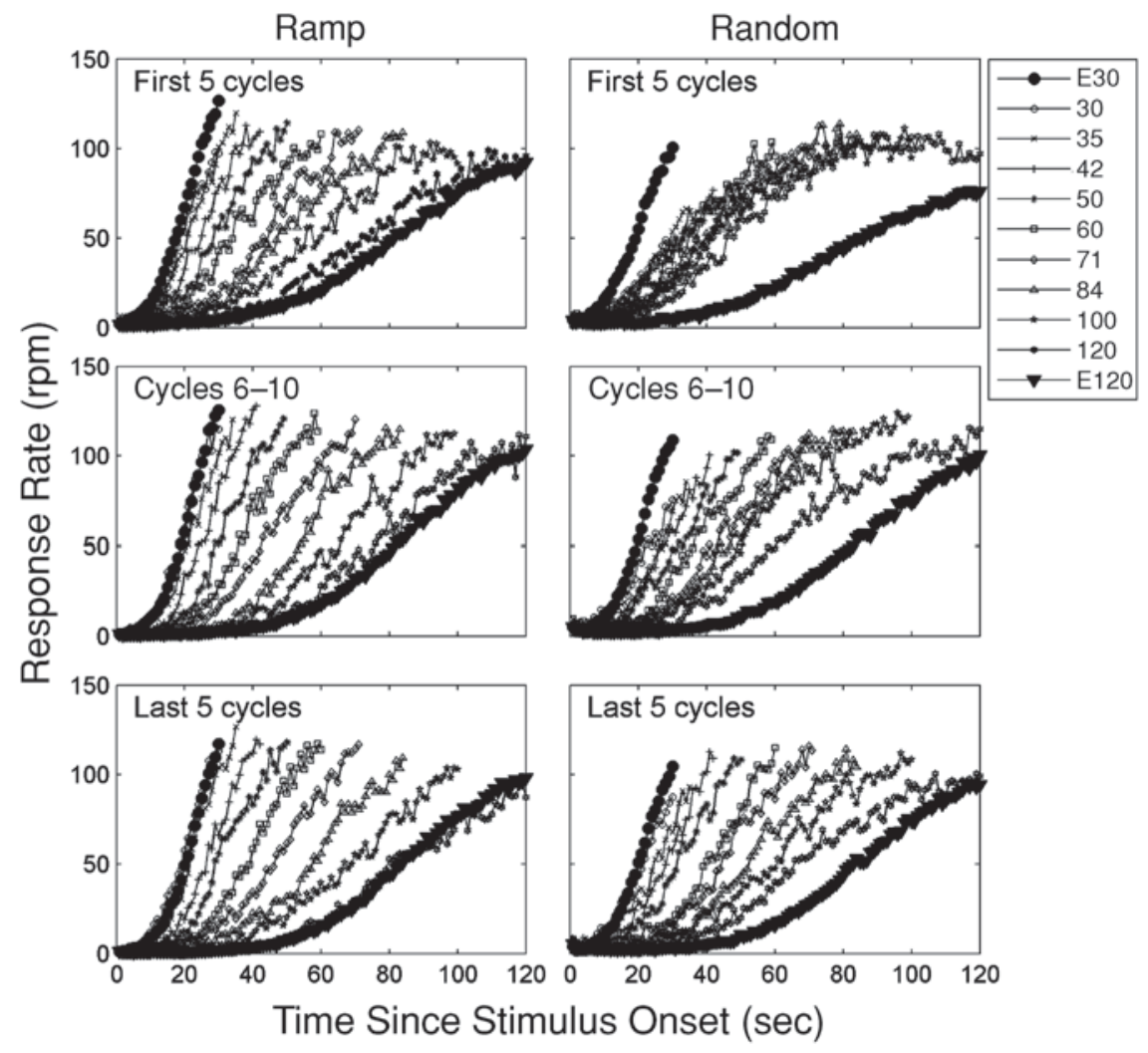

Figure 6. Response rate as a function of time since stimulus onset for rats in the ramp condition (left panels) and the random condition (right panels). These data are shown for the first five cycles (top panels), the next five cycles (middle panels), and the last five cycles (bottom panels). One stimulus remained on a fixed-interval schedule of $30 \mathrm{sec}$ (solid circles); another stimulus remained on a fixed-interval schedule of $120 \mathrm{sec}$ (solid triangle); the third stimulus changed daily among the nine intervals between 30 and 120 sec.

$30 \mathrm{sec}$ ) provided evidence for between-intervals temporal discrimination by comparing performance under intervals of different durations. Some measures (such as the time of the median response, the time of response rate change, and the temporal gradient parameters) provided evidence both of within-interval and between-intervals temporal learning.

\section{Comparison of the Acquisition of Stimulus and Temporal Discriminations}

In the present data, acquisition of stimulus and withininterval temporal discrimination developed simultaneously, as was observed in Kirkpatrick and Church (2000a), rather than showing a pattern in which stimulus discrimination must precede temporal discrimination (Gallistel \& Gibbon, 2000, 2002). Although the speed of development of the stimulus discrimination ratio was slightly more rapid than that of the temporal discrimination ratio, both of these measures changed in a similar manner as a function of sessions. The temporal discrimination certainly did not begin after the stimulus discrimination was complete (bottom panel of Figure 2).
The speed and asymptote of learning of the stimulus and the temporal discrimination ratios were similar for different intervals. Although the discrimination ratios (stimulus discrimination ratio and temporal discrimination ratio) for the simultaneous training condition increased more rapidly than did those for the blocked training condition when plotted as a function of cycles, the functions were approximately the same when plotted as a function of sessions. This suggests that the discrimination ratio for one interval was increased by training on the other intervals. This is plausible because an initial low rate of responding is learned in all the FI conditions.

\section{Direct Predictions of Summary Measures of Temporal Discriminations}

An empirical approach to the identification of a learning curve that applies to many different summary measures of temporal discrimination is to identify a function form and then adjust the parameters of this function to fit the data. Exponential equations provided a good way to summarize the learning of each of the dependent measures of temporal discrimination. The dependent measures of perfor- 
mance described in this section and listed in Table 1 were examined as a function of amount of training.

The functions relating the dependent variables to the amount of training were all reasonably well fit by threeparameter exponential equations. The median $\omega^{2}$ was .98 (see Table 1). For each dependent variable, the exponential equation accounted for a high percentage of the variance of 30 data points, and the parameters of the equation were systematically related to the conditions of training. This suggests that these equations were reliable (i.e., repeatable) and that they provided a good description of each of the dependent variables (i.e., the residuals from the equation were small and, perhaps in some cases, nonsystematic).

The five measures of the acquisition of temporal discrimination had three different patterns. As a function of the FI $(30,60$, and $120 \mathrm{sec})$, two of them began and ended at different levels (time of median response and time of maximum rate change); two of them began at similar levels and ended at different levels (time of first response and rate at comparable intervals), and one of them began and ended at similar levels (temporal discrimination ratio). Of course, there are many additional summary measures that could be described. The acquisition of these are also likely to be fit by exponential equations, but it is not clear how to predict in advance which of the parameters for different conditions will be the same and which will be different.

A problem with the description of the dependent variables with exponential equations for the explanation of acquisition is that they are difficult to relate to an underlying learning process. If a single dependent variable were used, one might assume that the initial level of the exponential function represented the initial state of knowledge, the asymptote of the exponential function represented the final state of knowledge, and the rate of approach to the asymptote represented the speed of learning. But with the use of multiple dependent variables, it is necessary to explain why there are differences among the initial and final states of knowledge and the rates of learning of the different dependent variables. An explanation that requires different equations or parameters to fit different summary measures of behavior may be regarded as a good description of each dependent variable, but not as an explanation of the raw output data that consists of a time series of stimuli, responses, and reinforcements.

Of course, the limitation encountered when exponential equations are used to account for multiple dependent measures of learning may also apply when theoretical models of the process are used to account for different dependent measures. For example, stochastic models of learning with parameters for initial value, rate of learning, and asymptotic level could be used, but the basis for the use of different parameters for different dependent measures is unclear (Bush \& Mosteller, 1955). The value of such models is demonstrated when the same parameter values that account for the acquisition function also account for other dependent variables, as Bush and Mosteller have done in their analysis of an avoidance learning experiment by using the same parameter values to account for acqui- sition, mean number of trials before the first avoidance, and mean number of shocks (Table 11.8, p. 257). These problems may be avoided by assuming that there is only an ordinal relationship between predicted and summary measures of behaviors, but at the cost of a reduction in the precision of prediction (Rescorla \& Wagner, 1972).

\section{Acquisition of the Response Pattern}

The temporal gradients produced by many quantitative theories of timing may be approximated by ogival functions (see the top panels in Figure 4). These functions may superpose when plotted in relative scales: Relative time refers to the ratio of time since stimulus onset to the time from stimulus onset to food availability, and relative response rate refers to the ratio of response rate since stimulus onset to the maximum response rate (see the bottom panels in Figure 4). This superposition result has been used extensively for the development of scalar timing theory (Gallistel \& Gibbon, 2000; Gibbon, 1977, 1991; Gibbon \& Church, 1990; Gibbon, Church, \& Meck, 1984).

The relative response rate as a function of relative time since stimulus may be approximated by an ogive. The equation for an ogive with $y$ between 0 and 1 is

$$
y=1 /\left[1+\left(e^{-(t-c) / s}\right)\right],
$$

where $c$ is a measure of the center and $s$ is a measure of the scale. The equation was fit to the mean relative response rate for the blocked condition as a function of time since relative stimulus onset $(t)$ with a nonlinear search algorithm. This is the fitted line in the lower right panel of Figure 4 . The center $(c)$ was close to 0.67 , the scale $(s)$ was close to 0.125 , and the proportion of variance accounted for $\left(\omega^{2}\right)$ was .995 . The same function provided a good approximation to the response rate gradients at all intervals (Equation 4). This is known as the superposition result (see the bottom right panel in Figure 4).

This same fitted line, an ogive, is shown in the top panel of Figure 7 and is labeled as $\lambda$. The flat line labeled $\kappa$ is an operant level; in this example, it was set at 0.2.

The acquisition of the function that defines the pattern of responding on each cycle is given by the linear operator model in Equation 5B. In this equation, $p$ is the observed pattern (a vector) of responding during a session, $n$ is the cycle number, $\lambda$ is the observed asymptotic pattern (a vector), and $\alpha$ is a constant, normally between 0 and 1 :

$$
p_{0}=\kappa
$$

and

$$
p_{n}=p_{n-1}+\alpha\left(\lambda-p_{n-1}\right), \text { where } n>0 .
$$

This is the standard equation for a linear operator model in which $\alpha$ is usually considered to be a learning rate. The pattern on cycle $n$ is equal to the performance on the previous cycle plus a proportion of the difference between the asymptotic pattern $(\lambda)$ and the pattern on the previous cycle $\left(p_{n}\right)$. The terms in Equation 5B can be rewritten to the form shown in Equation 5C. In this form, the pattern 


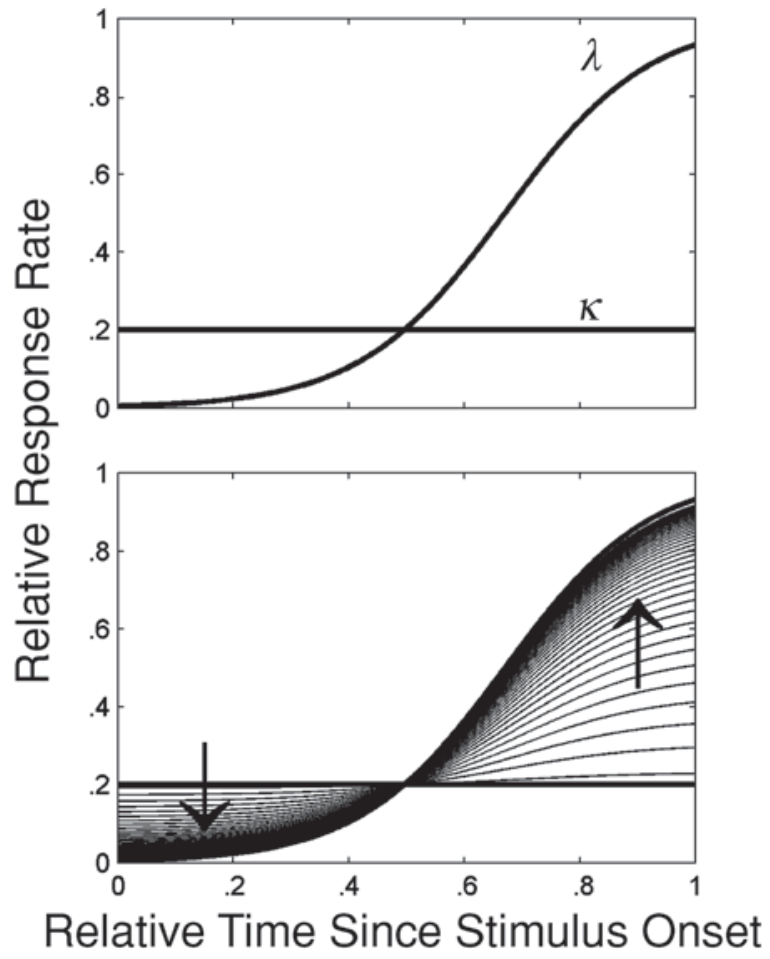

Figure 7. Acquisition of the response pattern. Top panel: An operant process consists of a mean level of responding that is constant throughout the interval $(\kappa)$ and a timing process that consists of an ogive that increases during the interval $(\lambda)$. Bottom panel: With training, the relative contribution of the timing process increases. The arrows show the directions of change with increased training.

on cycle $n$ is recognized to be a weighted average of the pattern on the previous cycle and the asymptotic pattern:

$$
p_{n}=\alpha p_{n-1}+(1-\alpha) \lambda, \text { where } n>0 .
$$

Thus, on the initial cycle, the pattern, $p_{0}$, will be a constant and, on subsequent cycles $(n)$, will approach the asymptotic values of the relative ogive $(\lambda)$. The rate of approach will be determined by the learning rate parameter, $\alpha$. The arrows indicate that beyond a fixed time, the relative response rate increases as a function of cycles and that, before that time, it decreases.

Because the same events occur on every cycle, the recursive form of Equations $5 \mathrm{~A}$ and $5 \mathrm{~B}$ can be rewritten as a linear equation (Bush \& Mosteller, 1955, pp. 58-61):

$$
p_{n}=\alpha^{n} \kappa+\left(1-\alpha^{n}\right) \lambda
$$

The relative operant level line, $\kappa$, and the relative ogive function, $\lambda$, were combined by this weighted averaging rule to produce the expected value of the pattern on any cycle. This linear form is particularly convenient because it makes it possible to generate the response pattern vector on each cycle directly from the values of $\alpha, n, \kappa$, and $\lambda$ and it does not require the prior calculation of $p_{n-1}$. The pattern changed with training: It equaled $\kappa$ initially and approached $\lambda$ with extensive training. Given a description of the initial behavior of $\kappa$ and $\lambda$, this general acquisition model requires only a single parameter, $\alpha$, to estimate the expected response pattern at any cycle $n$.

Any timing or conditioning theory that produces an approximately flat initial response gradient and an approximately ogival response gradient at asymptote may provide a quantitative account of the acquisition of the response pattern with the use of Equation 5D. For the prediction of the time of occurrence of responses, $p_{n}$ may be used as a probability of responding in some short interval of time (a response rate). The prediction of the response gradients, however, does not necessarily lead to a correct prediction of the response bouts or the various dependent measures shown in Figure 3.

\section{A Packet Theory of the Dynamics of Temporal Discrimination}

A packet theory of timing has been used to account for asymptotic performance of the response rate and many other dependent measures of performance in many different procedures (Kirkpatrick, 2002; Kirkpatrick \& Church, 2003). A slightly modified version of packet theory, referred to as Version 2, was applied to the asymptotic results of additional procedures (Church \& Guilhardi, 2005; Guilhardi, Keen, MacInnis, \& Church, 2005). The packet theory of the dynamics of temporal discrimination described here produces the same asymptotic results as Version 2 of packet theory, except that it has two modifications, which will be described later, that allow it to make reasonable predictions about the dynamics of temporal discrimination.

This process created simulated data that could be analyzed in the same way as the actual data.

The four parts of the model, labeled perception, memory, decision, and responses, are shown in Figure 8.

Perception. The perception was determined by the procedure, so there were no free parameters. At any given time between stimulus onset and reinforcement, a time to food may be calculated on the basis of the last stimulusto-food interval $(d)$ and the time elapsed from the onset of the last stimulus $(t)$. This is called the perception:

$$
s(t)=d-t, \quad t \leq d .
$$

Memory. Memory is a weighted sum of the perceived time to food and the remembered time to food. The weighted sum is described by the following standard linear equation:

$$
E_{n+1}(t)=\alpha s(t)+(1-\alpha) E_{n}(t),
$$

where $s(t)$ is the current perceived time to food, $E_{n}(t)$ is the current remembered time to food, $\alpha$ is the learning parameter, and $n$ is the current number of reinforcements. This linear equation was used by Bush and Mosteller (1955) to describe learning of the probability of a response, and it is used here to describe the learning of expected durations to reinforcement (a vector) as a function of physical time. 

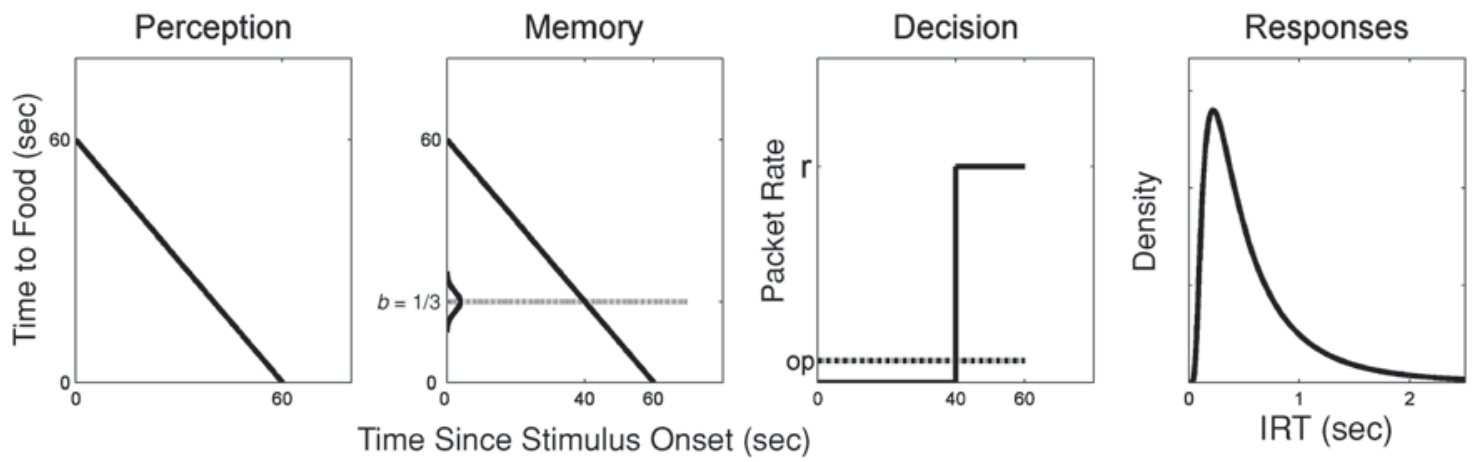

Figure 8. Packet theory: perceived time to food (Perception), remembered time to food (Memory), rate of packet initiation (Decision), and probability density of interresponse times (IRTs; Responses). See the text for details.

Memory was determined by a learning rate parameter $(\alpha=0.0125$ per cycle). The multiplication was the product of a scalar and a vector, and the addition was a sum of corresponding elements of two vectors (array calculations). The starting expected values of memory were determined by a normal distribution with a mean of $400 \mathrm{sec}$ and a standard deviation of $280 \mathrm{sec}$. The value of memory at time zero, $E(0)$, was used as the estimate of the mean remembered time of reinforcement at stimulus onset. (It was set to the mean of $400 \mathrm{sec}$ if the sample was below 0 .)

Decision. In packet theory, packets are initiated by a transformation of memory via a threshold and by a constant operant rate; these packets generate responses. The horizontal line in the memory panel of Figure 8 represents a sample of a threshold. The threshold transforms the continuous pattern in memory into a pattern with two states: a high state with rate $(r)$ of initiating packets of responses and a low state with no initiation of new packets of responses. In every cycle, a single random sample $(b)$ is taken from a normal distribution $(\eta)$, with a mean between zero and one $\left(\mu_{b}\right)$, and some coefficient of variation $\left(\gamma_{b}\right)$, as described in Equation 8:

$$
b=\eta\left(\mu_{b}, \gamma_{b}\right) \quad\left(0 \leq \mu_{b} \leq 1\right) .
$$

If the sample is below zero, $b$ is resampled, and if it is above one, it is set to one. Thus, $b$ is a proportion between zero and one. The threshold $B$ is defined in Equation 9:

$$
B=P_{b}[E(t)] \quad[0 \leq t \leq E(0)],
$$

where $P_{b}$ is the $b$ th percentile of the memory function $E(t)$ when $t$ is between zero and $E(0)$. The threshold $B$ is a time such that, when memory is above $B$, the decision function is in the low state, and when memory is below $B$, the decision function is in the high state $(r)$.

In addition, at all times, packets are generated at some operant rate $(o p)$. The total rate of anticipatory packet generation is $r+o p$. In the present simulation, the decision to initiate a packet of responses was determined by the normally distributed threshold distribution with a mean $\left(\mu_{b}\right)$ of .333 and a coefficient of variation $\left(\gamma_{b}\right)$ of 0.5 , by an operant rate $(o p)$ of 0.6 packets per minute, and by a function that related rate of packet initiation $(r)$ to the mean re- membered reinforcement interval at stimulus onset, $E(0)$, as described in Equation 10:

$$
r=-.30 \log _{10} E(0)+.92,
$$

where $E(0)$ is the mean remembered reinforcement interval at stimulus onset in seconds and $r$ is the number of packet initiations per minute. A new estimate was calculated after each delivery of food. Therefore, memory (at time 0 ) is the expected time to the next food that determines the mean response rate based on the linear relationship between rate and $\log$ (interval).

The two modifications of Version 2 of packet theory were related to the acquisition process: (1) The starting remembered expectation was as determined by a random sample, rather than being set at 0 , and (2) the estimated response rate of packet initiation $(r)$ was a function of the mean remembered time since stimulus onset on each cycle, rather than a constant function at all cycles.

Responses. The observed pattern of responding is often characterized by bouts of responses that occur with short interresponse times and that are separated by longer interbout intervals (Tolkamp \& Kyriazakis, 1999). One example is the head entry response of a rat in an appetitive classical or operant procedure (Kirkpatrick, 2002; Kirkpatrick \& Church, 2003). The term bout will be used for characteristics of an observed series of responses. For example, a bout may be described as a series of responses with no interresponse intervals greater than some criterion; the characteristics of bouts then may be described by the frequency distribution of interresponse intervals in a bout, the number of responses in a bout, and the duration of a bout (Kirkpatrick \& Church, 2003).

If a packet is initiated, the mean number of responses is determined by a Poisson distribution with a mean of 5 , and the interresponse times are determined by a Wald distribution with a location $(\mu)$ of $0.75 \mathrm{msec}$ and a scale

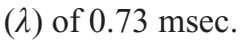

The term packet will be used as a theoretical term to refer to the characteristics of the response units generated by the model. A packet consists of a variable number of responses with variable interresponse times. The number of responses per packet was assumed to be a Poisson dis- 
tribution with a mean of five responses per packet; the interresponse times within a packet were positive values distributed as a Wald (inverse Gaussian) distribution, shown in the last panel of Figure 8 and described in Equation 11:

$$
f(t)=\left(\frac{\lambda}{2 \pi t^{3}}\right)^{\frac{1}{2}} \exp \left(\frac{-\lambda(t-\mu)^{2}}{2 \mu^{2} t}\right),
$$

where $\mu$ is the center parameter, $\lambda$ is the scale parameter, and $t$ is the interresponse time in seconds.

The simulations were based on the same procedures as those used in the experiment and on the same number of cycles as in the data. This process created simulated data that were analyzed in the same way as the actual data.

The simulated data made it possible to estimate the response gradients as a function of time since stimulus onset, as is shown in Figure 9. The gradients are shown for the $30-, 60-$, and 120-sec FI procedures for the first six sessions of training and for the last five sessions (top three panels). The bottom panel of the figure shows the asymptotic data (solid line) and the model estimates (thin lines).

The same simulated data as those used for estimation of the response gradients were also used for estimation of the dependent measures of performance on the temporal discrimination task. The same parameter values were used for all the panels in Figures 9 and 10. The dependent measures were the response gradient, the time of median response, the time of transition from the low to the high response rate, the time of first response, the response rate at comparable times, and the temporal discrimination ratio. As was noted previously, the functions relating the dependent variables to amount of training varied considerably. They were measured in different units (response time, response, and proportion). As can be observed in the data, some measures began and ended at different levels; some measures began at the same level and ended at different levels, and some measures began and ended at the same level. Despite the differences in units and the parameters needed for exponential fits, the process model generated data that were similar to the observed patterns. The $\omega^{2}$ measure is given for each dependent variable in the panels in Figures 9 and 10.

The same parameters as those used to simulate the initial acquisition were also used for the simulations of the daily transitions of the middle interval in both the ramp and the random conditions (except for the rate of learning, as will be described later in this section). In these simulations, the performance on each session began with the final performance on the previous session.

The simulated gradients (Figure 11) were similar to the observed gradients (Figure 6) in both the ramp and the random conditions. The variance accounted for, $\omega^{2}$, was .896. Although the gradients of responding with the ramp and random conditions were quite different in the first five cycles, this was due to the conditions of training on the previous session. (Note that in the ramp condition, the intermediate interval on the previous session was only
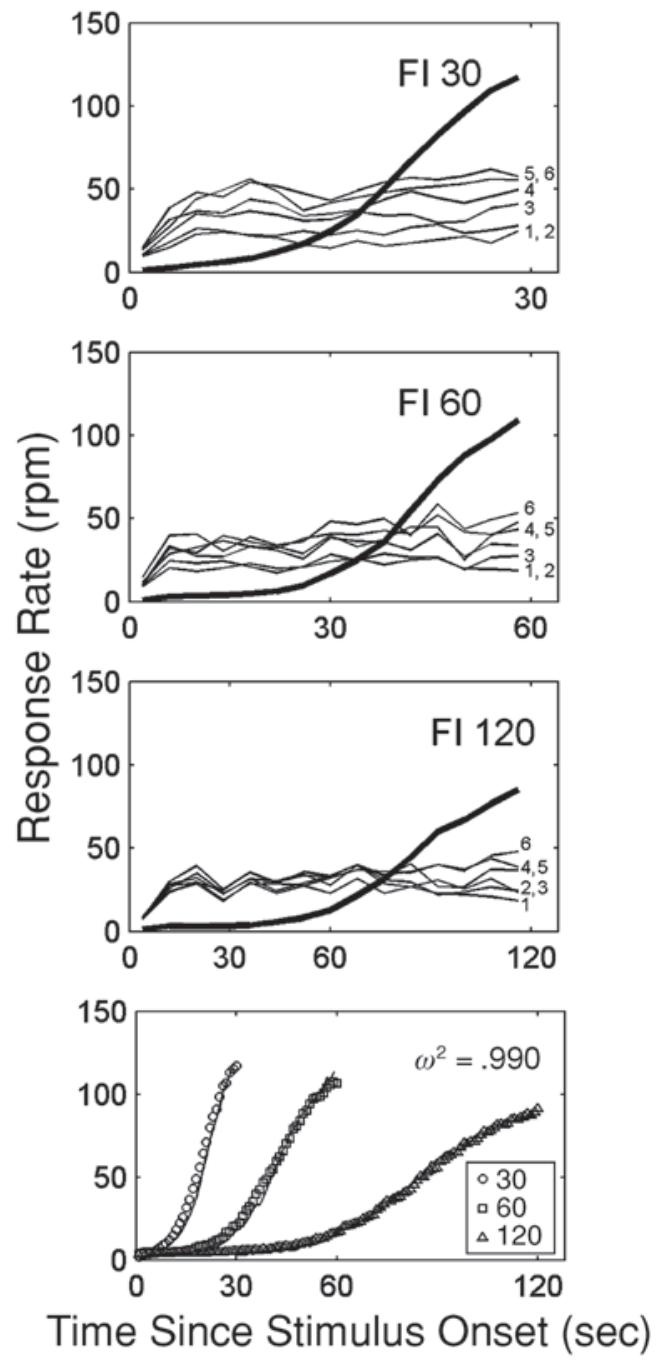

Figure 9. Simulation of response rate as a function of time since stimulus onset. The top three panels show the first six blocks of 20 cycles (labeled 1-6) and the last 15 blocks of 20 cycles (thick lines) for the blocked conditions with 30-, 60-, and 120-sec intervals, respectively. The bottom panel shows the response rate during the stimulus as a function of time since stimulus onset for the three intervals (solid points) and the simulated values (thin lines).

slightly shorter or longer than the intermediate interval on the current session.)

The same value of the learning rate $(\alpha)$ was used for the simulations of the ramp and random conditions shown in Figure 11. Because the same learning rate could be used for both conditions, the difference in observed response gradients was based on the treatment in the previous session, rather than on any differential speed of learning.

Although value of the learning rate $(\alpha)$ has only a negligible effect on asymptotic performance, it profoundly affects the development of the performance. The effect of variations in $\alpha$ on the temporal gradients is shown in Figure 12 for the three FI conditions (FI 30, FI 60, and FI $120 \mathrm{sec}$ ) as 

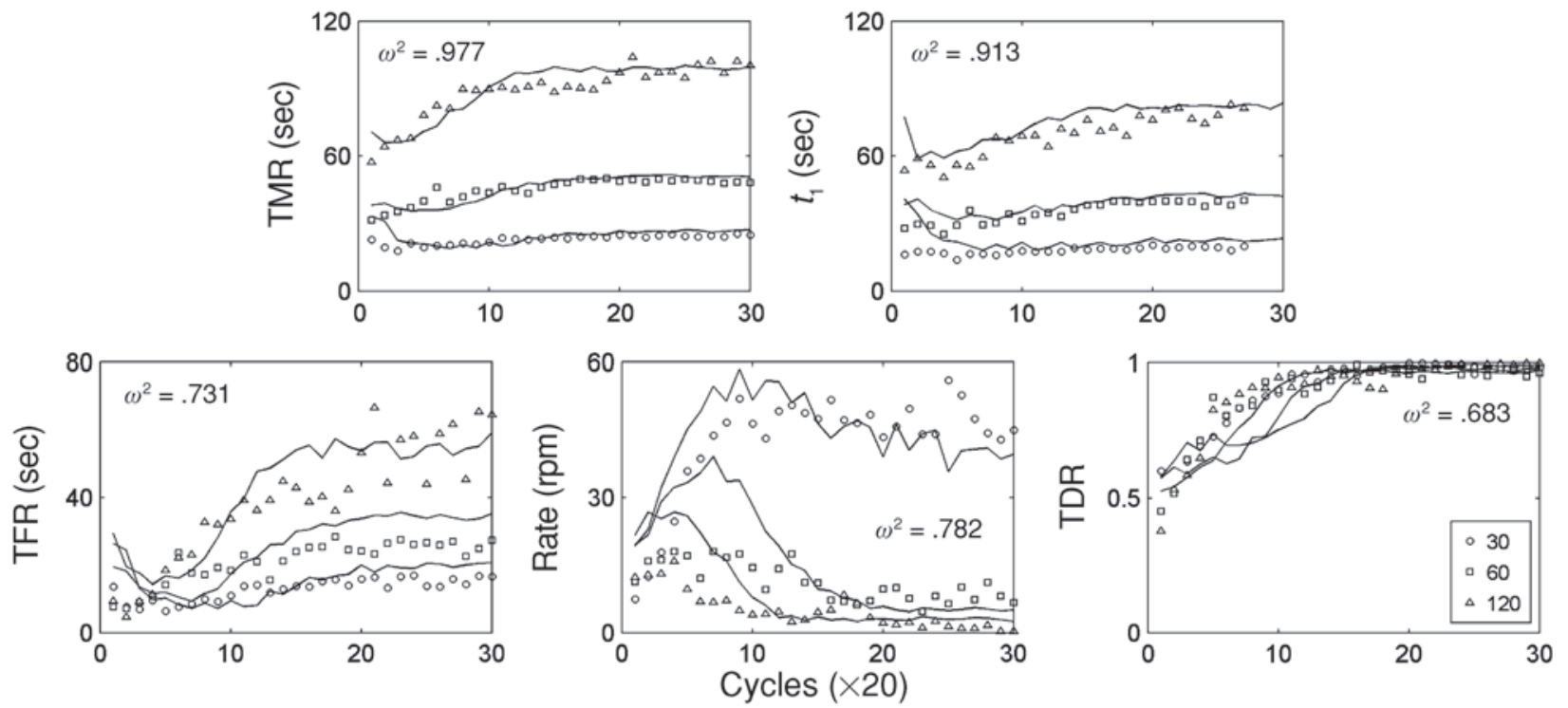

Figure 10. Simulation of five measures of the acquisition of temporal discriminations based on a model of temporal learning. The thin lines are based on the model of temporal learning with the same parameters as those used for the fitting of the gradients in Figure 9. The measures are the time of median response (TMR; first panel), the time of transition ( $t_{1}$; second panel), the time of first response (TFR; third panel), the mean response rate in responses per minute (fourth panel), and the temporal discrimination ratio (TDR; fifth panel).

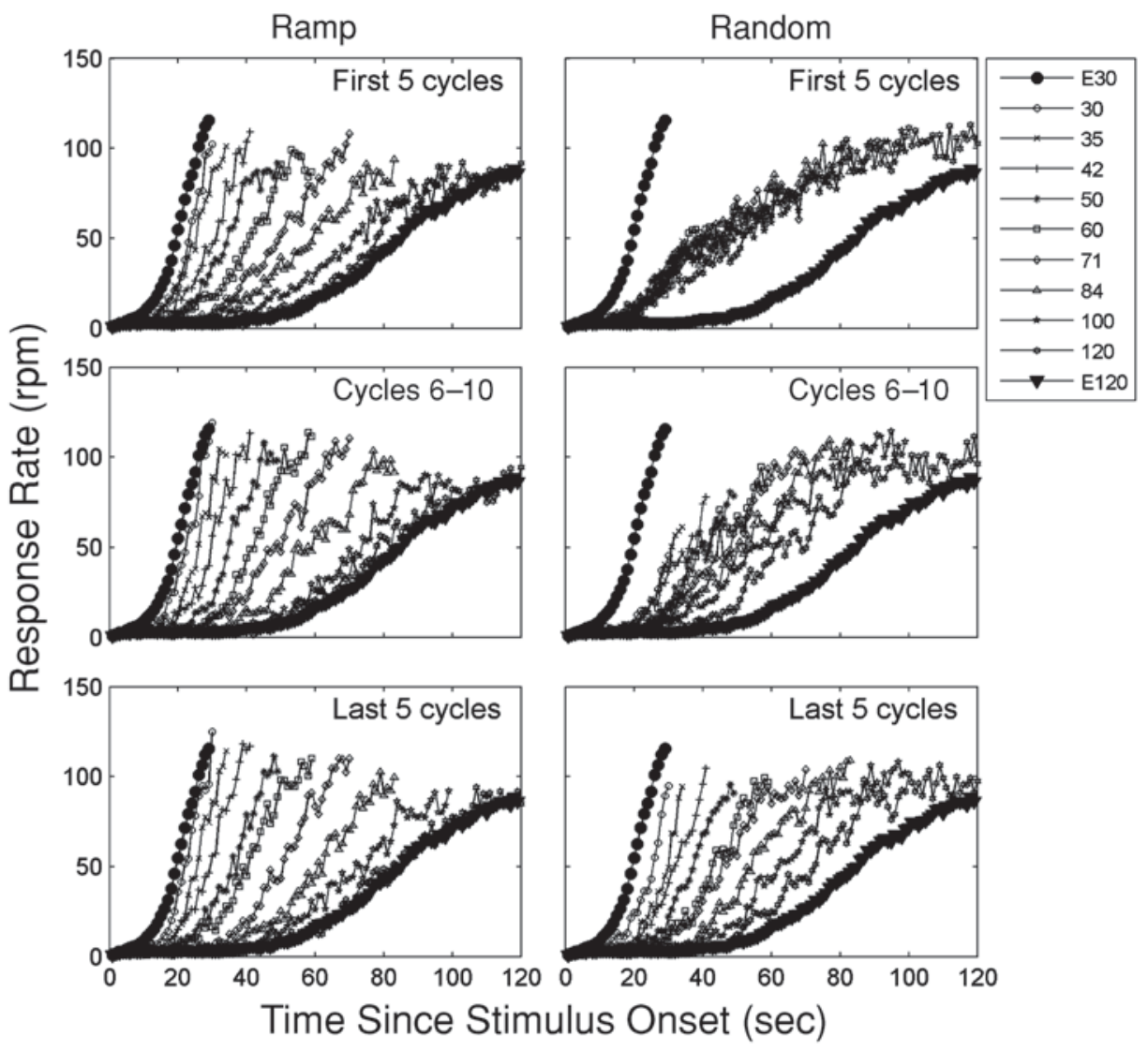

Figure 11. Predictions of the model of temporal learning for the adjustment of response rate to daily changes in the duration of the middle interval in the ramp condition (left panels) and the random condition (right panels). The first five cycles, the next five cycles, and the last five cycles of a session are shown in top, middle, and bottom panels, respectively. 
$\mathrm{FI} 30$
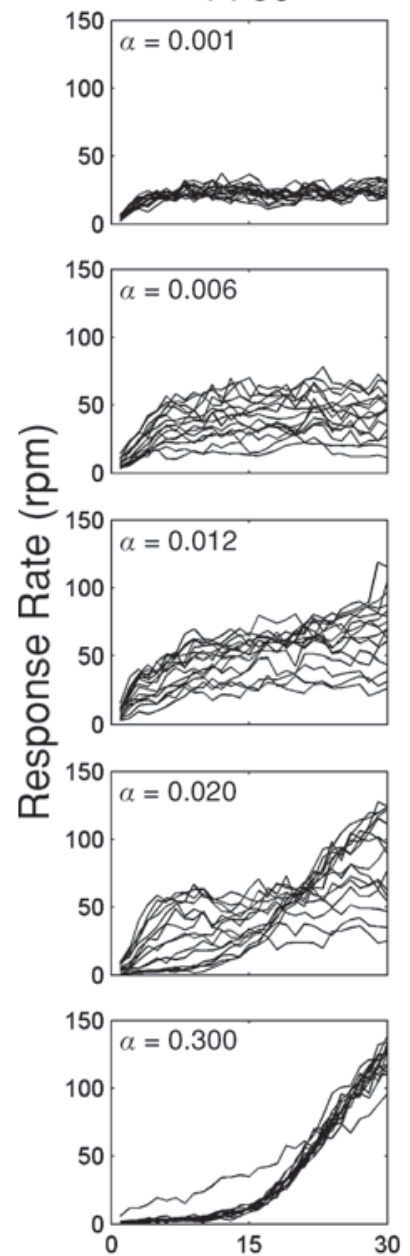

Time Since
$\mathrm{FI} 60$
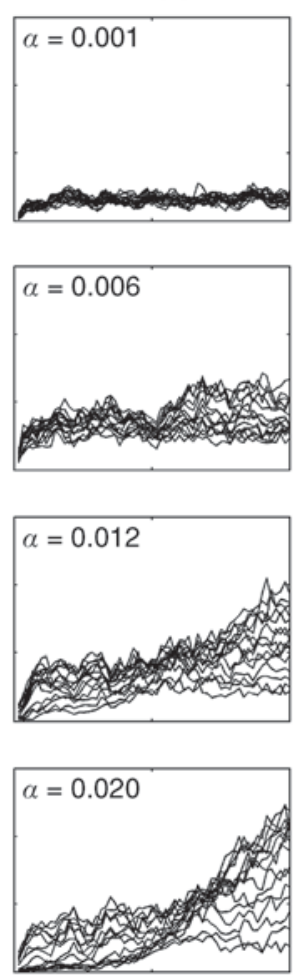

$\alpha=0.300$

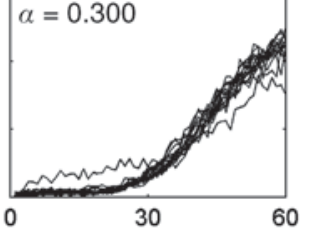

600
FI 120
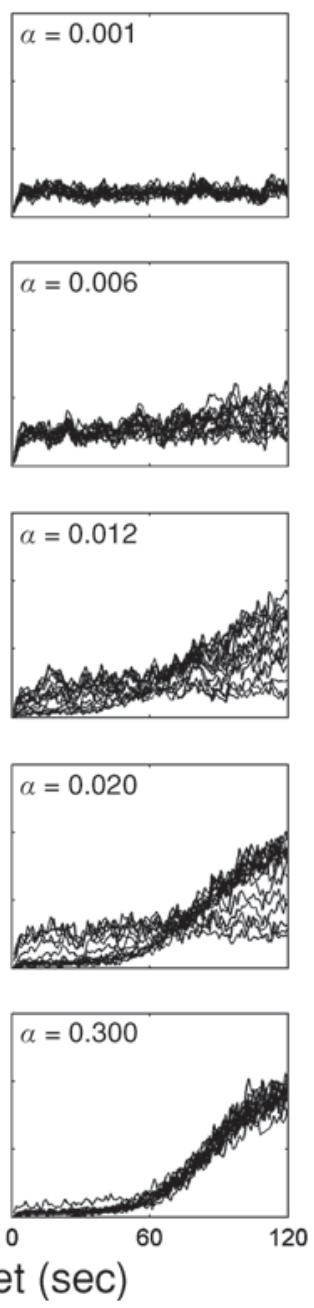

Figure 12. Mean simulated response rate as a function of stimulus onset. The columns display the results for the 30-, 60-, and 120-sec intervals; the rows display the different learning rates $(\alpha)$.

a function of blocks of 20 cycles. With $\alpha=0.001$, the gradients are relatively flat and constant as a function of training (top row of panels); with $\alpha=0.300$, the gradients are close to asymptotic after the first block of 20 cycles (bottom row of panels); with $\alpha=0.012$ (third row of panels), the gradients approach the asymptotic level at about the same rate as that in the data (Figure 5). The effects of learning rates intermediate between the optimal value and a value that is clearly too high or low are shown in the second and fourth rows of panels. A comparison between the development of the temporal gradients produced by the rats and the model can be calculated for any value of the learning rate. The percentage of variance accounted for $\left(\omega^{2}\right)$ was used as the measure of goodness of fit.

The relationship between the proportion of variance accounted for in goodness of fit and different values of the learning rate is shown in Figure 13. The top panel is based on the first 15 sessions of baseline training (Phase 1) for the simultaneous condition; the bottom panel is based upon the first 15 sessions of the conditioning with the daily changes in interval (Phase 2). Note that the scale of learning rates in the bottom panel is 10 times the scale in the top panel. The best estimate of the learning rates are $\alpha=0.012$ for the top panel and $\alpha=0.101$ for the bottom panel. In the original learning, the function relating the goodness of fit to the learning rate has a clear maximum slightly above 0.01 ; with the daily transitions, the maximum of the function is clearly much higher than 0.01 , but it can only be roughly identified at about 0.10 . In the ramp condition, one cannot rule out learning rates that are substantially higher, but the simplest assumption is that they were the same in both conditions but that it was not possible to obtain reliable measures of the speed of learning for a procedure in which there were only small daily changes in the FI.

The substantially faster learning shown in the bottom panel may have been a result of the procedure or of the 

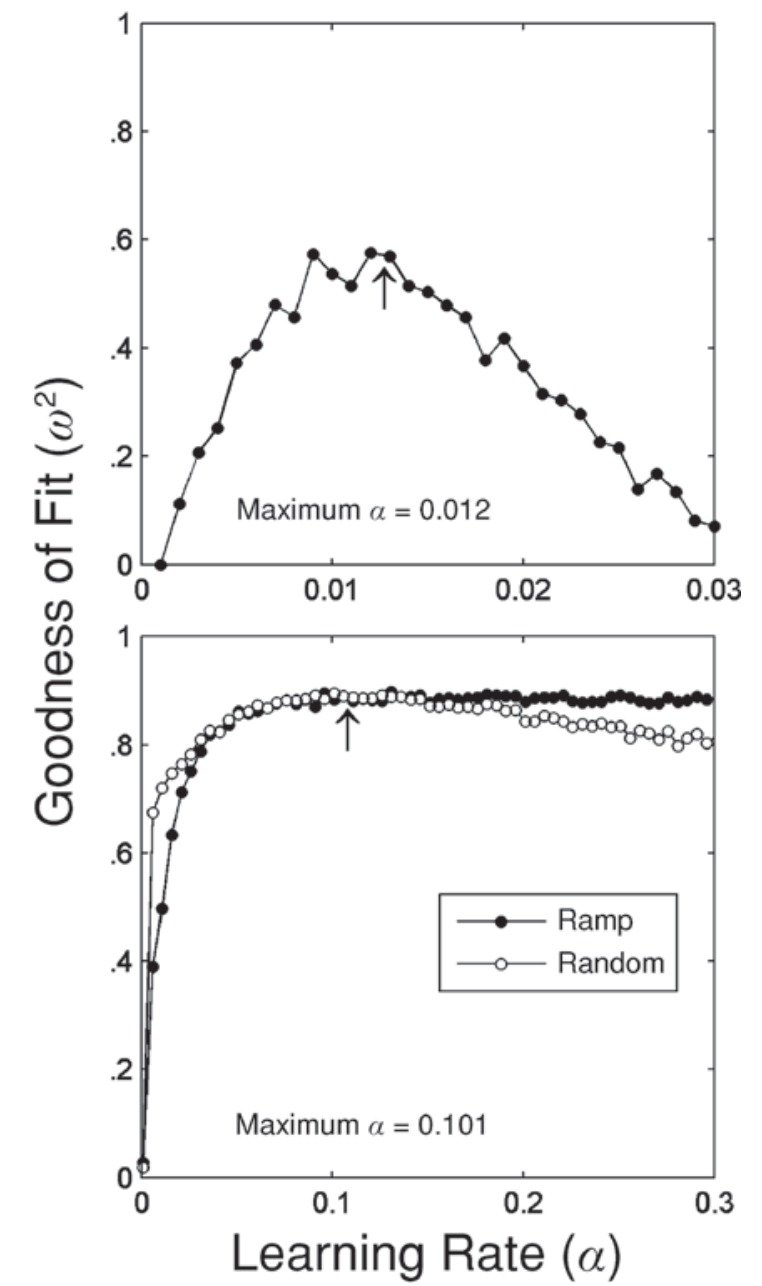

Figure 13. The mean goodness of fit $\left(\omega^{2}\right)$ as a function of learning rate $(a)$. The top panel displays the goodness of fit of the model to the data of the first half of baseline training (Phase 1). The bottom panel displays the goodness of fit of the model to the data for all 36 sessions of the daily changed intervals (Phase 2). Note that the learning rate scale and the best estimate of the learning rate are about 10 times higher in the bottom panel than in the top panel.

additional temporal discrimination training. Estimates of learning rate become more unstable as a function of training, but no systematic increase in the learning rate was observed within either phase. The first acquisition of stable temporal discriminations may be fundamentally different from the acquisition of daily changes in a temporal interval. Although the results of both procedures appear to be due to the same processes, further analysis will be required to understand why the learning rate is 10 times faster under one procedure than under the other.

\section{Direct Empirical and Theoretical Approaches}

In the direct approach, the goal is to describe and/or explain the behavioral dependent variables individually.

A direct empirical approach. With a direct approach, (1) the conditions of a procedure are selected, (2) one or more summary dependent variables are selected, (3) functional relationships are established between the conditions of the procedure and the values of the dependent variables, (4) the functional relationships are described quantitatively, and (5) the functional relationships are categorized into principles. For example, in the study of temporal discrimination, (1) an FI schedule of reinforcement may be selected as the procedure, (2) the dependent variable may be the mean response rate as a function of time since the last reinforcement, (3) this dependent variable may increase as a function of time since the last reinforcement, (4) the increase may be characterized by a simple equation, and (5) the similarities of the equations for different intervals may lead to a general principle.

This is a standard empirical approach. With this approach, Dews (1970) found that the function relating the rate of the keypecking response of pigeons (expressed as a proportion of the maximum response rate) to the time since last reinforcement (expressed as a proportion of the time between reinforcements) was the same at very different intervals $(30,300$, and 3,000 sec). This observed superposition of functions is now referred to as timescale invariance.

If a quantitative fit of a model to experimental data is provided, it is usually applied only to a single summary statistic in any particular experiment; this makes it unclear whether or not the theory applies to other summary measures (Church \& Kirkpatrick, 2001). Some of the principles of timing, such as proportionality, the scalar principle, Weber's law, and timescale invariance, may apply to some dependent measures but not to others (Zeiler \& Powell, 1994). Unless the equations for different dependent variables can be derived from each other or from some more general formulation, the analysis must be regarded as descriptive curve fitting and not as explanatory modeling.

A direct theoretical approach. With a direct theoretical approach, (1) a model of the process that transforms the procedure into the behavior is proposed, (2) the conditions of one or more procedures are selected, (3) one or more summary dependent variables are selected, and (4) the values of the dependent variables generated by the animal are compared with the values of the dependent variables generated by the model.

This is a standard theoretical approach used in quantitative theories of timing and conditioning, such as scalar timing theory (Gibbon et al., 1984), the learning-to-time model (Machado, 1997), the multiple time scale model (Staddon \& Higa, 1999), and the temporal difference model (Sutton \& Barto, 1990). (See Church \& Kirkpatrick, 2001, for the application of these and other models to two dependent variables in a single procedure.) Although the standard theoretical approach may be used for explaining results, the generalizability of its predictions to different dependent measures and procedures is often not explicitly demonstrated.

\section{An Indirect Theoretical Approach}

In the indirect theoretical approach, the goal is to develop a process model that predicts time of occurrence of 
responses from which any behavioral dependent variable can be calculated. With this approach, (1) a model of the process that transforms the procedure into the behavior is proposed, (2) the conditions of one or more procedures are selected, (3) the time of occurrence of stimuli, responses, and reinforcers are simulated on the basis of the procedure and the model, (4) many dependent variables are defined, and (5) the values of the dependent variables generated by the animal are compared with the values of the dependent variables generated by the model.

With this approach, Kirkpatrick and Church (2003) found that many different measures of response rate and response pattern of head entry responses into a food cup by rats in fixed and random schedules of reinforcement could be simulated with a simple model of the process that was referred to as packet theory. An essential feature of this indirect approach is that, given a procedure within its domain, the model predicts the time of occurrence of responses. A good model of the process (combined with the procedure) will predict any summary dependent variable based on the raw data (times of occurrence of stimuli, responses, and reinforcers). Unlike the standard direct theoretical approach, in which a model of the process predicts some selected behavioral dependent variables, an indirect theoretical approach is a model of that process that predicts the times of occurrence of all responses. From this predicted raw data, any dependent measure of performance can be compared with the same dependent measured based on the observed raw data.

\section{Conclusions}

A direct approach to the description and explanation of the acquisition of a temporal discrimination is to identify one or more measures of temporal discrimination. Exponential equations provided a good description of the acquisition of five measures of temporal discrimination, but this was not an explanation of the behavior, because the parameters of these equations did not generalize across measures (dependent variables) or procedures.

An indirect approach to the description and explanation of the acquisition of a temporal discrimination is to identify the process of acquisition of a temporal discrimination. This makes it possible to generate simulated data sets that may be used for simulated measures of temporal dependent measures that can be compared with the observed data. A packet theory of FI responding provided a good description of the five measures of temporal discrimination, and the same model generalized across measures (dependent variables) and procedures.

Acquisition of temporal discriminations may involve several general processes: the perception of a time interval, memory of reinforced time intervals, decision about responding, and emission of packets of responses. The same general processes that account for asymptotic temporal discriminations may also account for the acquisition of temporal discriminations if a learning rule is added. A standard linear operator model provides an excellent approximation to this learning rule.

\section{REFERENCES}

Bush, R. R., \& Mosteller, F. (1955). Stochastic models for learning. New York: Wiley.

Catania, A. C. (1970). Reinforcement schedules and psychophysical judgments: A study of some temporal properties of behavior. In W. N. Schoenfeld (Ed.), The theory of reinforcement schedules (pp. 1-42). New York: Appleton-Century-Crofts.

Catania, A. C., \& Reynolds, G. S. (1968). A quantitative analysis of the responding maintained by interval schedules of reinforcement. Journal of the Experimental Analysis of Behavior, 11, 327-383.

Church, R. M. (2002). The effective use of secondary data. Learning \& Motivation, 33, 32-45.

Church, R. M., \& Guilhardi, P. (2005). A Turing test of a timing theory. Behavioural Processes, 69, 45-58.

Church, R. M., \& Kirkpatrick, K. (2001). Theories of conditioning and timing. In R. R. Mowrer \& S. B. Klein (Eds.), Handbook of contemporary learning theories (pp. 211-254). Mahwah, NJ: Erlbaum.

Church, R. M., Meck, W. H., \& Gibbon, J. (1994). Application of scalar timing theory to individual trials. Journal of Experimental Psychology: Animal Behavior Processes, 20, 135-155.

Cumming, W. W., \& Schoenfeld, W. N. (1958). Behavior under extended exposure to a high-value fixed interval reinforcement schedule. Journal of the Experimental Analysis of Behavior, 1, 245-263.

DEWs, P. B. (1970). The theory of fixed-interval responding. In W. N. Schoenfeld (Ed.), The theory of reinforcement schedules (pp. 43-61). New York: Appleton-Century-Crofts.

Dukich, T. D., \& LEE, A. E. (1973). A comparison of measures of responding under fixed-interval schedules. Journal of the Experimental Analysis of Behavior, 20, 281-290.

FERSter, C. B., \& SKInNER, B. F. (1957). Schedules of reinforcement. New York: Appleton-Century-Crofts.

Fry, W., Kelleher, R. T., \& CoOK, L. (1960). A mathematical index of performance on fixed-interval schedules of reinforcement. Journal of the Experimental Analysis of Behavior, 3, 193-199.

Gallistel, C. R., \& Gibbon, J. (2000). Time, rate and conditioning. Psychological Review, 107, 289-344.

Gallistel, C. R., \& Gibbon, J. (2002). The symbolic foundations of conditioned behavior. Mahwah, NJ: Erlbaum.

Gallistel, C. R., Mark, T. A., King, A. P., \& Latham, P. E. (2001). The rat approximates an ideal detector of changes in rates of rewards: Implications for the law of effect. Journal of Experimental Psychology: Animal Behavior Processes, 27, 354-372.

GibBon, J. (1977). Scalar expectancy theory and Weber's law in animal timing. Psychological Review, 84, 279-325.

Gibbon, J. (1991). Origins of scalar timing theory. Learning \& Motivation, 22, 3-38.

Gibbon, J., \& Church, R. M. (1990). Representation of time. Cognition, 37, 23-54.

Gibbon, J., Church, R. M., \& Meck, W. H. (1984). Scalar timing in memory. In J. Gibbon \& L. Allan (Eds.), Timing and time perception (Annals of the New York Academy of Sciences, Vol. 423, pp. 52-77). New York: New York Academy of Sciences.

Gollub, L. R. (1964). The relations among measures of performance on fixed-interval schedules. Journal of the Experimental Analysis of Behavior, 7, 337-343.

GuIlHardi, P., \& CHURCh, R. M. (2004). Measures of temporal discrimination in fixed-interval performance: A case study in archiving data. Behavior Research Methods, Instruments, \& Computers, 36, 661-669.

Guilhardi, P., Keen, R., Macinnis, M. L. M., \& Church, R. M. (2005). The combination rule for multiple intervals. Behavioural Processes, 69, 189-205

Higa, J. J. (1997). Rapid timing of a single interfood interval duration by rats. Animal Learning \& Behavior, 25, 177-184.

Higa, J. J., Thaw, J. M., \& Staddon, J. E. R. (1993). Pigeons' wait-time responses to transitions in interfood-interval duration: Another look at cyclic schedule performance. Journal of the Experimental Analysis of Behavior, 59, 529-541.

InNIS, N. K., \& STADDON, J. E. (1971). Temporal tracking on cyclicinterval reinforcement schedules. Journal of the Experimental Analysis of Behavior, 16, 411-423. 
KirkPatrick, K. (2002). Packet theory of conditioning and timing. Behavioural Processes, 57, 89-106.

KirKPATRICK, K., \& ChURCH, R. M. (2000a). Independent effects of stimulus and cycle duration in conditioning: The role of timing processes. Animal Learning \& Behavior, 28, 373-388.

KirkPatrick, K., \& ChURCh, R. M. (2000b). Stimulus and temporal cues in classical conditioning. Journal of Experimental Psychology: Animal Behavior Processes, 26, 206-219.

KirKPATRICK, K., \& CHURCH, R. M. (2003). Tracking of the expected time to reinforcement in temporal conditioning procedures. Learning \& Behavior, 31, 3-21.

Lejeune, H., Ferrara, A., Simons, F., \& Wearden, J. H. (1997). Adjusting to changes in the time of reinforcement: Peak interval transitions in rats. Journal of Experimental Psychology: Animal Behavior Processes, 23, 211-231.

Machado, A. (1997). Learning the temporal dynamics of behavior. Psychological Review, 104, 241-265.

Machado, A., \& CeviK, M. (1998). Acquisition and extinction under periodic reinforcement. Behavioural Processes, 44, 237-262.

MaCKIntosh, N. J. (1974). The psychology of animal learning. London: Academic Press.

Meck, W. H., Komeily-Zadeh, F. N., \& Church, R. M. (1984). Two step acquisition: Modification of an internal clock's criterion. Journal of Experimental Psychology: Animal Behavior Processes, 10, 297-306.

Rescorla, R. A., \& Wagner, A. R. (1972). A theory of Pavlovian conditioning: Variations in the effectiveness of reinforcement and nonreinforcement. In A. H. Black \& W. F. Prokasy (Eds.), Classical conditioning II: Current research and theory (pp. 64-99). New York: Appleton-Century-Crofts.

RoBeRTS, S. (1981). Isolation of an internal clock. Journal of Experimental Psychology: Animal Behavior Processes, 7, 242-268.

SCHNEIDER, B. A. (1969). A two-state analysis of fixed-interval responding in the pigeon. Journal of the Experimental Analysis of Behavior, 12, 677-687.

Skinner, B. F. (1938). The behavior of organisms: An experimental analysis. New York: Appleton-Century-Crofts.

Spence, K. W. (1956). Behavior theory and conditioning. New Haven, CT: Yale University Press.

Staddon, J. E. R., \& Higa, J. J. (1999). Time and memory: Toward a pacemaker-free theory of interval timing. Journal of the Experimental Analysis of Behavior, 71, 215-251.

Sutton, R. S., \& Barto, A. G. (1990). Time-derivative models of Pavlovian reinforcement. In M. Gabriel \& J. Moore (Eds.), Learning and computational neuroscience: Foundations of adaptive networks (pp. 497-537). Cambridge, MA: MIT Press.

TATHAM, T. A., \& ZURN, K. R. (1989). The MED-PC experimental apparatus programming system. Behavior Research Methods, Instruments, \& Computers, 21, 294-302.

Tolkamp, B. J., \& KYriazAKIs, I. (1999). To split behaviour into bouts, log-transform the intervals. Animal Behaviour, 57, 807-817.

Zeiler, M. D., \& Powell, D. G. (1994). Temporal control in fixedinterval schedules. Journal of the Experimental Analysis of Behavior, 61, 1-9.

\section{ARCHIVED MATERIALS}

The following materials associated with this article may be accessed through the Psychonomic Society's Norms, Stimuli, and Data archive, http://www.psychonomic.org/archive/.

To access these files or links, search the archive for this article using the journal (Learning \& Behavior), the first author's name (Guilhardi) and the publication year (2005).

FILE: Guilhardi-L\&B-2005.zip.

DESCRIPTION: The compressed archive file contains:

One readme file (readme.pdf). The readme file contains a description of the supplementary material such as content, file formats, and file naming conventions.

Data files $(1,584)$ for each of the 66 sessions for each of the 24 rats. The primary data are the times (column 1) of events (column 2) that occurred during the experimental session, such as the times of responses, food deliveries, and onset and termination of stimuli. Some analysis tools (MATLAB source code) are available as supplementary material from Guilhardi \& Church (2004).

LiNK: http://www.brown.edu/Research/Timelab.

DESCRIPTION: Contains additional data in the same format, documentation of the procedures and formats, and references to publications that analyzed aspects of these additional data.

AUTHORS’E-MAIL ADDRESSES: paulo_guilhardi@brown.edu, russell _church@brown.edu.

(Manuscript received April 12, 2004; revision accepted for publication February 11, 2005.) 